ARTICLE

Received 24 Nov 2015 | Accepted 22 Feb 2016 | Published 15 Apr 2016

DOl: 10.1038/ncomms11121

OPEN

\title{
ZBTB2O is required for anterior pituitary development and lactotrope specification
}

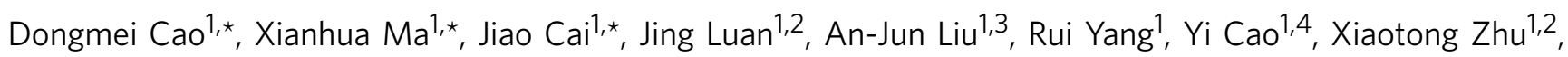
Hai Zhang ${ }^{1}$, Yu-Xia Chen ${ }^{1}$, Yuguang Shi ${ }^{5}$, Guang-Xia Shi ${ }^{2}$, Dajin Zou ${ }^{4}$, Xuetao Cao ${ }^{6}$, Michael J. Grusby ${ }^{7}$, Zhifang $X^{1} e^{1,3} \&$ Weiping J. Zhang ${ }^{1}$

The anterior pituitary harbours five distinct hormone-producing cell types, and their cellular differentiation is a highly regulated and coordinated process. Here we show that ZBTB2O is essential for anterior pituitary development and lactotrope specification in mice. In anterior pituitary, ZBTB2O is highly expressed by all the mature endocrine cell types, and to some less extent by somatolactotropes, the precursors of prolactin (PRL)-producing lactotropes. Disruption of Zbtb2O leads to anterior pituitary hypoplasia, hypopituitary dwarfism and a complete loss of mature lactotropes. In ZBTB20-null mice, although lactotrope lineage commitment is normally initiated, somatolactotropes exhibit profound defects in lineage specification and expansion. Furthermore, endogenous ZBTB2O protein binds to Prl promoter, and its knockdown decreases PRL expression and secretion in a lactotrope cell line MMQ. In addition, ZBTB2O overexpression enhances the transcriptional activity of Prl promoter in vitro. In conclusion, our findings point to ZBTB2O as a critical regulator of anterior pituitary development and lactotrope specification.

\footnotetext{
${ }^{1}$ Department of Pathophysiology, Second Military Medical University, 800 Xiangyin Road, Shanghai 200433, China. ${ }^{2}$ Department of Pathophysiology, Dalian Medical University, 9 West Section, Lvshun South Road, Dalian, 116044, China. ${ }^{3}$ Department of Cell Biology, Second Military Medical University, 800 Xiangyin Road, Shanghai 200433, China. ${ }^{4}$ Department of Endocrinology, Changhai Hospital, 168 Changhai Road, Shanghai 200433, China.

${ }^{5}$ Barshop Institute for Longevity and Aging Studies, University of Texas Health Science Center at San Antonio, 15355 Lambda Drive, San Antonio, Texas 78245, USA. ${ }^{6}$ National Key Laboratory of Molecular Biology and Department of Immunology, Chinese Academy of Medical Sciences, 9 Dongdan Santiao, Beijing, 100005, China. 7 Department of Immunology and Infectious Diseases, Harvard School of Public Health, 651 Huntington Avenue, Boston, Massachusetts, 02115, USA. * These authors contributed equally to this work. Correspondence and requests for materials should be addressed to W.Z. (email: wzhang@smmu.edu.cn) or to Z.X. (email: xiezf@smmu.edu.cn).
} 
T he pituitary gland functions as a relay between hypothalamus to peripheral target organs and plays a central role in regulating homeostasis, metabolism, growth, reproduction and lactation ${ }^{1,2}$. It is composed of two anatomically and functionally different entities, the adenohypophysis, including the anterior and intermediate lobes, and neurohypophysis, also known as the posterior lobe. The anterior lobe harbours five distinct specialized cell types defined by trophic hormones they generate and secrete. Somatotropes produce growth hormone $(\mathrm{GH})$; lactotropes secrete prolactin (PRL); thyrotropes generate thyroid stimulating hormone (TSH); gonadotropes produce luteinizing hormone (LH) and follicle-stimulating hormone (FSH); and corticotropes secrete adrenocorticotropin hormone (ACTH).

Pituitary organogenesis is a complexly regulated and highly coordinated process both in time and space. While the neurohypophysis is derived from the neural ectoderm, the adenohypophysis originates from Rathke's pouch, which is an invagination of the oral ectodermin response to neural epithelium signals $^{2}$. The proliferative progenitors in definitive Rathke's pouch undergo gradual differentiation and migration, and eventaully give rise to the five endocrine cell types in the anterior pituitary at birth. During early postnatal life, the anterior lobe expands rapidly, mainly caused by the stimulation of cell proliferation by trophic hormones from hypothalamus. In the developing pituitary, many transcription factors are temporally and spatially regulated, and critically involved in the lineage specification and differentiation ${ }^{2}$.For example, Pitx1, Pitx2, Lhx3, Prop1 and Pax6 control the early phases of pituitary patterning, while Pit-1 (Pou1f1), SF1, Gata2 and Tbx19 regulate cell differentiation ${ }^{1,3}$. It has been reported embryonic stem cells can be stimulated by these factors to differentiate into various endocrine cells in vitro ${ }^{4}$, while mutations in these transcription factors have been linked with congenital hypopituitarism and several combined or isolated pituitary hormone deficiencies. Particularly, Pit-1 is required for the differentiation of three lineages: thyrotropes, somatotropes and lactotropes. Pit-1 expression begins at embryonic day 13.5 (E13.5) in mice and maintains in thyrotropes, somatotropes and lactotropes throughout adulthood. In a cell-specific manner, Pit-1 regulates the transcription of genes encoding hormone products, such as $\mathrm{TSH}, \mathrm{GH}, \mathrm{PRL}$ and growth hormone-releasing hormone receptor (GHRHR $)^{1,5-7}$. Therefore, mutations with the Pit 1 gene are associated with combined GH, PRL and TSH deficiency ${ }^{8-10}$.

The ontogeny of lactotropes is closely related with somatotrope lineage, and highly regulated by hormones and growth factors. Lactotropes are scarce in the fetal anterior pituitary, and begin to increase at postnatal day 3 (P3) in mice, following the appearance of GH-expressing cells. Notably, the early lactotropes also express $\mathrm{GH}$ in the rat and cow ${ }^{11}$. Importantly, a majority of lactotropes are depleted in the transgenic mice using rat GH promoter to target the destruction of GH-expressing cells ${ }^{12,13}$, thus establishing a notion that a majorty of lactotropes arise from the dual hormone-secreting somatolactotropes. However, how lactotropes are specified from somatolactotropes is still an enigma. There are some evidence that oestrogen, insulin-like growth factor 1 (IGF1) and fibroblast growth factor 2 (FGF2) regulate the cell proliferation and differentiation of lactotroptes, but none of these factors are essential for lactotrope specification. Treatment of fetal pituitary with $17 \beta$-estradiol in vitro increases the number of PRL-positive cells ${ }^{14}$. Disruption of either oestrogen receptor alpha gene (Era) or Igf1 leads to a marked reduction both in PRL expression and lactotrope cell number, however, with no obvious effects on lactotrope specification ${ }^{15-17}$. Although FGF2 is capable of initiating differentiation of lactotropes rather than somatotropes from a Pit1-expressing progenitors in vitro ${ }^{18}$, no obvious pituitary phenotypes have been reported in $F g f 2$ knockout mice ${ }^{19-21}$. Therefore, the regulatory mechanism about lactotrope development remains unclear.

ZBTB20 belongs to a subfamily of zinc finger proteins containing $\mathrm{C} 2 \mathrm{H} 2$ Krüppel-type zinc fingers and $\mathrm{BTB} / \mathrm{POZ}$ domain $^{22}$. It plays important roles in multiple systems, as suggested by the severe phenotypes of the mice lacking ZBTB20, which mainly include growth retardation, premature lethality, infertility and hypoglycemia ${ }^{23-26}$. Noteworthy, many of the null phenotypes implicate a possibility of hypopituitarism, however, little is known about its role in pituitary biology. In this study, we show that ZBTB20 is highly expressed by all the mature endocrine cell types in anterior pituitary, and its deficiency leads to anterior pituitary hypoplasia and hypopituitarism as a result of complete absence of mature lactotropes and impaired expansion of somatotropes. ZBTB20-null mice display sever defects in lactotrope specification and expansion despite the normal initiation of its lineage commitment, whereas the mice lacking Zbtb20 specifically in nervous system show normal lactotrope development. ZBTB20 knockdown in MMQ lactotrope cells results in a robust decrease in PRL expression and secretion. Furthermore, we demonstrate that ZBTB20 binds to $\mathrm{Prl}$ promoter and enhances its transcriptional activity. Thus our findings for the first time establish a critical role of ZBTB20 in anterior pituitary development and lactotropic lineage specification.

\section{Results}

Hypopituitarism in ZBTB20-deficient mice. To evaluate the potential role of ZBTB20 in pituitary biology, we first examined pituitary development and function in ZBTB20-null mice. As previously described ${ }^{24}, Z b t b 20$ global knockout mice were grossly indistinguishable from their control littermates at birth, but exhibited significant postnatal growth retardation, premature mortality and infertility, which are indicative of hypopituitarism. At the mixed genetic background of C57BL/6J and $129 \mathrm{~Sv}$, more than half of homozygous null mutants can survive up to 4 weeks, with a few to adulthood ${ }^{24}$. However, after backcrossed to C57BL/6J mice for 12 generations, a vast majority of mutant homozygotes died within 4 weeks of age. Therefore, we phenotypically analysed the mutant mice mainly within 3 weeks of age, when they were relatively healthy, however, with a dramatic reduction in body size and weight compared with littermate controls (Fig. 1a). At birth, there was no gross difference of pituitary between mutant and control littermates (Supplementary Fig. 1). From 1 week after birth, the mutant pituitaries were markedly smaller in size than littermate controls, thus they were only $\sim 50 \%$ of the normal weight at postnatal day 21 (P21; Fig. 1b). Of note, the hypoplasia was mainly observed in the anterior pituitaries from both male and female mutant mice, while their posterior lobes were not significantly affected. In addition, the female mutants also displayed profound hypoplasia in ovary and uterus (Fig. 1c). Whole mount of mammary glands revealed a severely impeded ductal elongation in 4-week-old null female mice, while age-matched control mice displayed extensive ductal elongation and branching (Fig. 1d). The hypothalamus that control pituitary development and function was anatomically and histologically normal in mutant mice. Taken together, the above observed growth retardation, sexual infantilism and anterior pituitary hypoplasia strongly suggest that ZBTB20 disruption results in hypopituitary dwarfism.

To assess GH deficiency in the mutants, we measured liver and serum levels of IGF-1, which are GH-dependent, given that random circulating levels of $\mathrm{GH}$ do not accurately reflect $\mathrm{GH}$ sufficiency due to the pulsatile nature of its secretion ${ }^{27}$. Compared with control mice, the mutants showed an $\sim 50 \%$ decrease in both liver messenger RNA (mRNA) and serum 
a

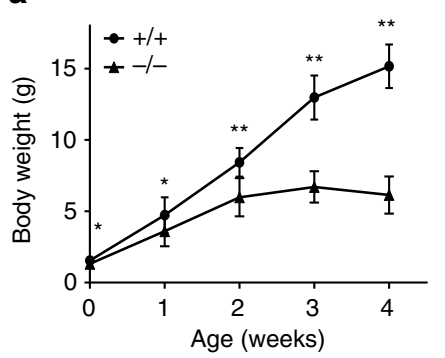

b

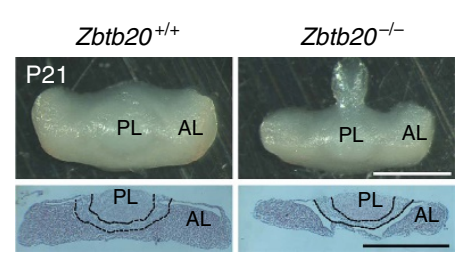

C

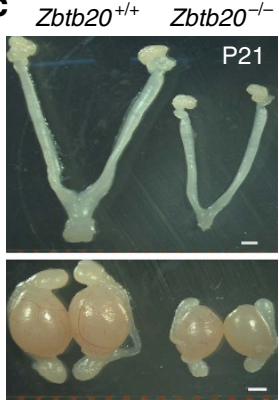

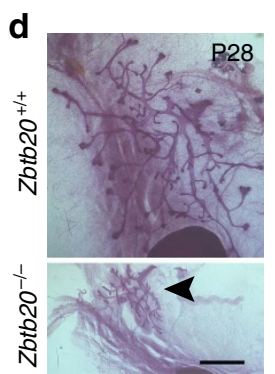

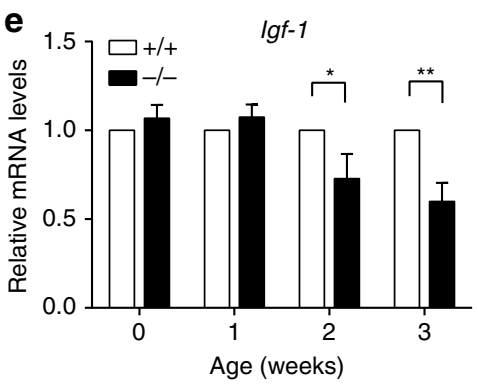

f

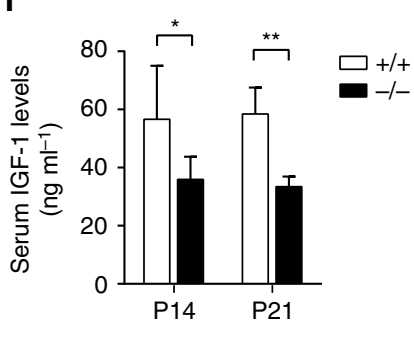

Figure 1 | Hypopituitarism in global ZBTB20-null mice. (a) Body weight of Zbtb20 ${ }^{+/+}$and Zbtb20- /- mice. $n=11-20$ per time point. Values represent mean \pm s.e.m. ${ }^{\star} P<0.05$ versus $+/+,{ }^{\star \star} P<0.01$ versus $+/+$ (Student's $t$-test.) (b) A general view (upper) and section (lower, H\&E) of 21-day-old

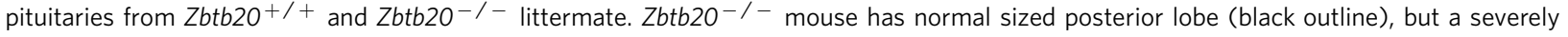
reduced anterior lobe.PL, posterior lobe of pituitary; $\mathrm{AL}$, anterior lobe. Scale bar, $1 \mathrm{~mm}$. (c) Uterus and ovaries from female mice (upper) and testes and epididymides from male mice (lower) are significantly reduced in 21-day-old $Z$ btb20-/ - mice compared with wild-type controls. Scale bar, $1 \mathrm{~mm}$.

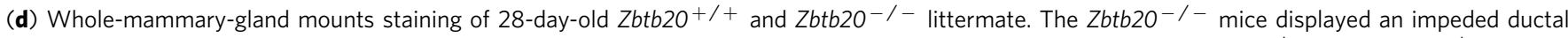
elongation (black arrowhead). Scale bar, $1 \mathrm{~mm}$. (e) Quantitative RT-PCR analysis of Igf- $1 \mathrm{mRNA}$ level in liver from $Z b t b 20^{+/+}$and $Z$ btb20 $0^{-/-}$mice. Results are presented as fold induction relative to mRNA level in wild-type mice. $n=3$ per time point. Values represent mean \pm s.e.m. ${ }^{\star} P<0.05,{ }^{\star \star} P<0.01$ (Student's $t$-test). (f) Circulating IGF-1 levels are detected by ELISA at P14 and P21. $n=6$ per time point. Values represent mean \pm s.e.m. ${ }^{\star} P<0.05$, ${ }^{\star \star} P<0.01$ (Student's $t$-test).

protein levels of IGF-1 at P21 (Fig. 1e,f). Combined with the anterior pituitary hypoplasia, these data indicate that ZBTB20-null mice at least have GH deficiency.

Impaired anterior pituitary development in ZBTB20-null mice. To determine the specific deficits in the mutant pituitary, we examined the alteration of its cell composition. Hematoxylin and eosin (H\&E) staining revealed a marked decrease in the number of granule-filled acidophilic cells (somatotropes and lactotropes) in the mutant pituitary compared with control at P21 (Supplementary Fig. 2), while basophilic cells (thyrotrophs, adrenocorticotrophs and gonadotrophs) were morphologically normal. We then performed immunohistochemical staining using antibodies specifically against individual hormone produced by each cell type. At P21, there were no apparent differences in the cell densities of GH-, TSH-, LH- or ACTH-immunoreactive cells between mutant and control mice (Fig. 2a). Very strikingly, PRL-immunoreactive cells were completely absent in mutant pituitary at the same age, in contrast to their abundant distribution in normal anterior lobe. The absence of mature lactotropes in mutant pituitary was further confirmed by immunostaining with the antibodies against dopamine receptor D2 (Drd2; Fig. 2b), which is another differentiation marker of lactotropes ${ }^{28,29}$. Consistently, quantitative reverse transcription PCR (RT-PCR) and western blotting analysis revealed that PRL expression was undetectable at either mRNA or protein levels in mutant pituitary at P14 (Fig. 2c,d). In addition, GH expression was decreased by $\sim 50 \%$ in mutant pituitary at both mRNA and protein levels compared to control, POMC mRNA levels increased by 1.46 -fold, while $L H$ and FSH mRNA levels were similar between both groups at P14 (Fig. 2c). Furthermore, measurement of the hormone contents in whole pituitary extracts by ELISA revealed that GH contents were decreased by $30 \%$ in mutants, PRL contents almost undetected, and TSH, ACTH and $\mathrm{LH}$ contents were not altered at P21 (Fig. 2e). These data suggest that ZBTB20 disruption selectively leads to a severe developmental defect of lactotropes, and to some less extent, somatotropes are affected as well.

ZBTB20 is essential for lactotrope lineage specification. To further characterize the developmental defect of lactotrope lineage in ZBTB20-null mice, we examined its ontogeny during embryonic and early postnatal periods. The lactotrope lineage emerges at E15.5 in mice, characterized by the expression of PRL in a restricted medial zone adjacent to the ventral surface of the intermediate lobe ${ }^{2}$, and undergoes rapid expansion during P7 to P21. Thus we first detected PRL expression in the developing pituitary. Consistently, immunohistochemical staining revealed that PRL-reactive cells were not detected at all in the mutant pituitary from P4 to P7 (Fig. 3a; Supplementary Fig. 3a). In addition, $\mathrm{Prl}$ mRNA expression was hardly detectable by RT-PCR after P2 (Supplementary Fig. 3b). Surprisingly, sparse PRLimmunoreactive cells were transiently detected in mutant pituitaries from E15.5 to birth, with no significant difference compared to control group (Fig. 3a; Supplementary Fig. 4). Moreover, $\mathrm{Prl}$ mRNA expression was also detected in mutant pituitary at birth, although the levels were decreased by $\sim 70 \%$ compared with control (Supplementary Fig. 3b). These data suggest that lactotrope lineage could be transiently initiated in Zbtb20-null pituitary in the perinatal period.

Given the crucial role of dual hormone-expressing somatolactotrope progenitors in lactotrope development ${ }^{13}$, we then analysed the generation and differentiation of somatolactotropes by double immunostaining of GH and PRL. At E15.5 and E16.5, 
a

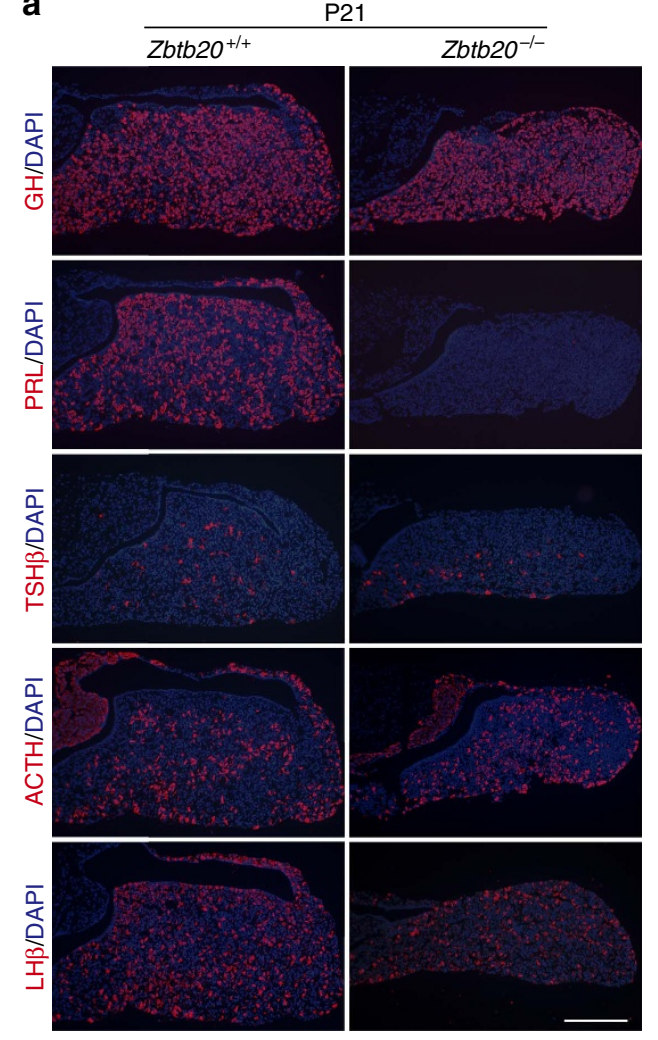

b

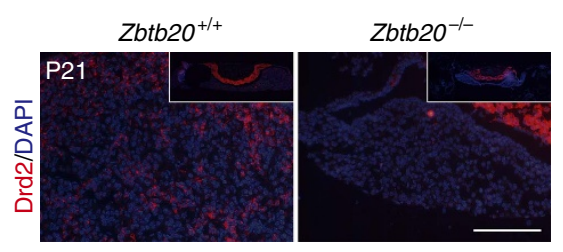

C

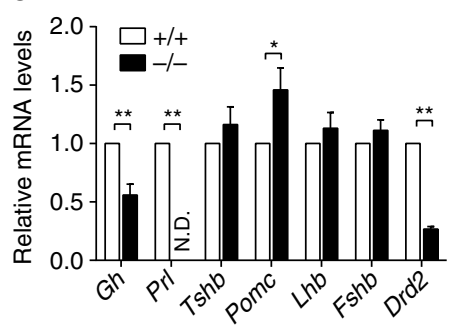

d
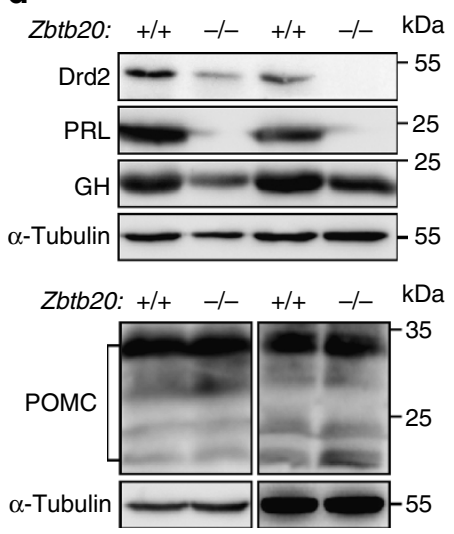

e

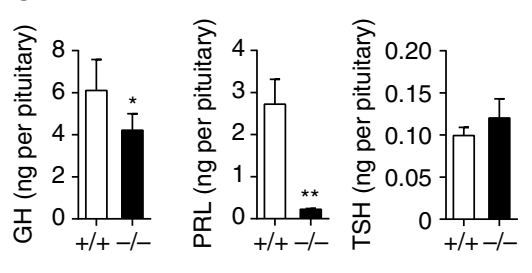

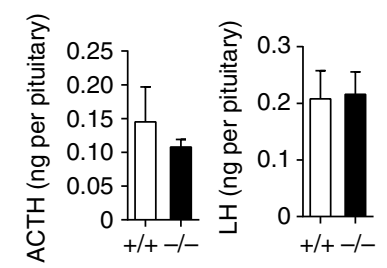

Figure 2 | Impaired development of endocrine cells in the anterior pituitary of Zbtb20-null mice. (a) All five types of cell lineages in anterior pituitary are detected by immunohistochemical staining using GH, PRL, TSH $\beta$, ACTH and LH $\beta$ antibodies in 21-day-old $Z$ btb20 $/ /+$ and $Z$ btb20-/- littermate. PRL-positive cells are absent in null mice, but other cell lineages have no apparent changes. (b) Immunohistochemical staining of Drd2 in 21-day-old pituitary sections also shows a loss of positive cells in the null mice. Insets show the whole coronal section of pituitaries. Scale bar, (a) $200 \mu \mathrm{m}$ and (b) $100 \mu \mathrm{m}$. (c) Quantification of hormone mRNA levels in 14-day-old Zbtb20 $0^{+/+}$and $Z$ btb20-/- mice by real-time RT-PCR. Results are presented as fold induction relative to mRNA levels in wild-type mice. A sample was pooled from three to five adenohypophyses of the same phenotype, and the experiment was repeated three times. Values represent mean \pm s.e.m. ${ }^{\star} P<0.05$, ${ }^{\star \star} P<0.01$ (Student's $t$-test). (d) Western blot of hormones and Drd2 proteins in pituitary extracts from 14-day-old $\mathrm{Zbtb} 2 \mathrm{O}^{+/+}$and $\mathrm{Zbtb20^{-/- }}$ mice. A sample was pooled from five to eight adenohypophyses of the same phenotype, and the experiment was repeated two times. (e) Anterior pituitary hormonal contents are measured in pituitary extracts by ELISA at P21. $n=3$. Values represent mean \pm s.e.m. ${ }^{\star} P<0.05$ versus $+/+,{ }^{\star \star} P<0.01$ versus $+/+$ (Student's $t$-test).

just like control mice, nearly all of PRL-expressing cells were GH-positive in mutant pituitaries, implying the apparently normal generation of somatolactotropes in the absence of ZBTB20 (Fig. 3a; Supplementary Fig. 4). Notably, in control pituitary, PRL single-positive cells $\left(\mathrm{PRL}^{+} \mathrm{GH}^{-}\right)$, which represent the specified lactotropes, began to emerge at E18.5, and gradually increased in the number and percentage to PRL-positive cells as the pituitary developed from $\mathrm{P} 0$ to $\mathrm{P} 14$. As a result, $\mathrm{PRL}^{+} \mathrm{GH}^{-}$ lactotropes account for over 75\% of PRL-positive cells at P4 and $\mathrm{P} 7$, while double-positive cells $\left(\mathrm{GH}^{+} \mathrm{PRL}^{+}\right)<25 \%$ (Fig. 3b,c). At P14, the vast majority of PRL-positive cells $(>95 \%)$ were $\mathrm{PRL}^{+} \mathrm{GH}^{-}$, and only a few $(<5 \%)$ were double-positive cells. In contrast, the mutant PRL-positive cells were consistently double-positive somatolactotropes from E15.5 to P0 (Fig. 3a,b; Supplementary Fig. 4), and completely vanished after P3, while $\mathrm{PRL}^{+} \mathrm{GH}^{-}$cells were not detected in the mutant pituitary during the early development. These data suggest that ZBTB20 may be not required for the initial commitment of somatolactotropes, but is indispensable for their specification into lactotropes.

Impaired cell proliferation in ZBTB20-null anterior pituitary. To investigate the cellular basis of pituitary hypoplasia in ZBTB20-null mice, we detected cell apoptosis and proliferation in postnatal pituitary. TUNEL staining revealed that very rare TUNEL-positive apoptotic cells were detected in the developing pituitaries from P7 to P21, with no significant difference between control and mutant mice (Supplementary Fig. 5). Then the proliferation rate was evaluated by measuring bromodeoxyuridine (BrdU) incorporation after a single intraperitoneal injection. There was no significant difference of BrdU incorporation into anterior pituitary cells between the two genotypes at E18.5 or P0 (Supplementary Fig. 6a). However, in comparison with control, the density of BrdU-positive cells was reduced in the mutant anterior pituitary by $\sim 27 \%$ ( $n=31-34$ slices from five animals) at P7 and P14, and more dramatically, by about $67 \%(n=27$ slices from four animals, $P<0.01$, Student's $t$-test) at P21 (Fig. 4a), indicating a defect of cell proliferation in the mutant pituitary.

To further characterize the cell proliferation of lactotropes and somatotropes in the developing pituitary, we did double immunostaining with anti-BrdU and anti-GH or anti-PRL antibodies, respectively. During P7 to P21, when the mutant pituitary were devoid of PRL-positive cells, double staining of BrdU and PRL revealed that the control mice exhibited efficient BrdU incorporation in PRL-positive cells, indicative of robust lactotrope lineage expansion (Fig. 4b). In addition, Zbtb20 deficiency also resulted in profound decrease in BrdU incorporation of somatotropes in the same period (Fig. 4c; Supplementary 
a
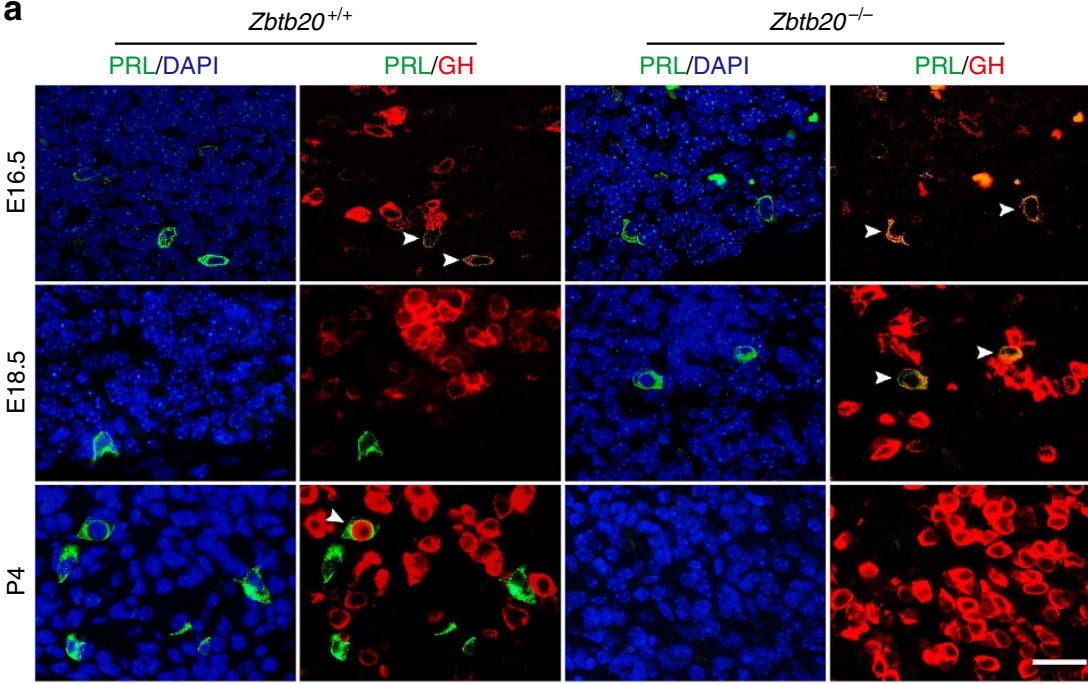

b
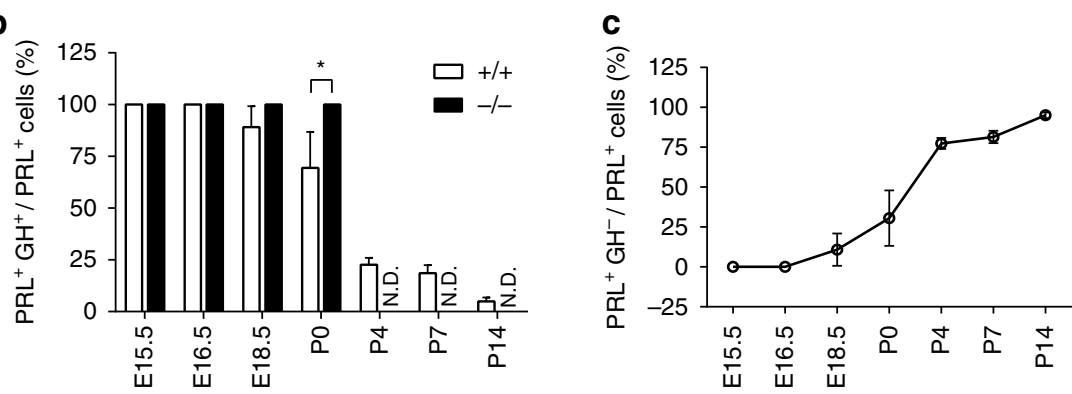

Figure 3 | Defect of lactotropes specification and differentiation in the absence of ZBTB20. (a) Immunohistochemical double staining of PRL (green) and GH (red) in the pituitaries from Zbtb20 ${ }^{+/+}$and $Z$ btb20 ${ }^{-/-}$mice at the age from E16.5 to P4. Cell nuclei were stained with DAPI (blue). Arrowheads indicate PRL and GH double-positive cells. Scale bar, $25 \mu \mathrm{m}$. (b,c) The percentages of double-positive cells (b) and PRL single-positive cells (c) among the PRL-positive cells in the pituitaries of wild-type mice are shown by diagram. E15.5: $n=28$ sections from four animals; E16.5: $n=23$ sections from five animals; E18.5: $n=15$ sections from four animals; PO: $n=21$ sections from five animals; P4: $n=26$ sections from five 5 animals; P7: $n=24$ sections from four animals; and P14: $n=13$ sections from two animals. Values represent mean \pm s.e.m. ${ }^{\star} P<0.05$ versus control (Student's $t$-test).

Fig. 6b). These data strongly indicate that pituitary hypoplasia in ZBTB20-null mice is primarily caused by the loss of lactotrope lineage development and the impairment of somatotrope expansion as well.

During postnatal development, the expansion of anterior pituitary originates from a proliferation of both already differentiated endocrine cells and stem/progenitor cells in the stimulation of proliferative signals. To determine whether the impaired cell proliferation was due to the defect of postnatal pituitary progenitors, we detected Sox 2 expression, which is a marker of pituitary progenitors ${ }^{30,31}$. Immunohistochemical analysis revealed that the number and distribution of Sox2positive cells were not significantly affected in anterior pituitary at P21 by the loss of ZBTB20 (Supplementary Fig. 7). These data suggest that the impaired cell proliferation in ZBTB20-null pituitary is not caused by the deficit of postnatal anterior pituitary progenitors, rather than the defect of differentiated endocrine cells.

Defects of lactotropic lineage expansion in ZBTB20-null mice. To evaluate the defect of lactotrope lineage expansion in the absence of ZBTB20 in more detail, we characterized somatolactotrope proliferation by triple immunostaining with the antibodies against GH, PRL and the cell proliferation marker Ki67. Unexpectedly, Ki67 expression was not detected in $\mathrm{GH}^{+} / \mathrm{PRL}^{+}$ cells from control mice until P4 (Fig. 5a,b), indicating a non-replicating state before $\mathrm{P} 3$. The Ki67-positive replicating somatolactotropes accounted for $6.5 \%$ (10/152) and $32.6 \%$ (30/92) of total $\mathrm{GH}^{+} / \mathrm{PRL}^{+}$cells at P4 and P7 (Fig. 5c), respectively. Interestingly, Ki67 protein was also present in $\mathrm{PRL}^{+} \mathrm{GH}^{-}$cells in control pituitary at P7 and P14 (Fig. 5b). These data suggest that both somatolactotropes and differentiated lactotropes are normally proliferative, and may contribute to the lactotrope lineage expansion in pituitary development. In contrast, ZBTB20-null somatolactotropes were consistently Ki67-negative, which transiently emerged before P3, and afterwards completely vanished before the entry into the time window of cell replication. Collectively, these data suggest that ZBTB20 is essential for the lineage specification and expansion of lactotropes.

Characterization of Pit-1 expression in ZBTB20-null pituitary. Considering the pivotal role of Pit-1 in the lineage development of somatotropes and lactotropes, we then analysed Pit-1 expression in the mutant pituitary. RT-PCR and in situ hybridization analyses revealed that Pit-1 mRNA expression levels were comparable between control and mutant pituitary at P0 and P2 (Fig. 6a; Supplementary Fig. 8a), and immunohistochemical staining showed no significant difference of Pit-1 protein expression between the two groups at E18.5 (Supplementary Fig. $8 \mathrm{~b}$ ).These data suggest that Pit-1 expression is not altered in the perinatal pituitary by the loss of ZBTB20. However, RT-PCR and western blot analyses revealed that ZBTB20 deletion led to a marked reduction in Pit-1 expression at both mRNA and protein 
a
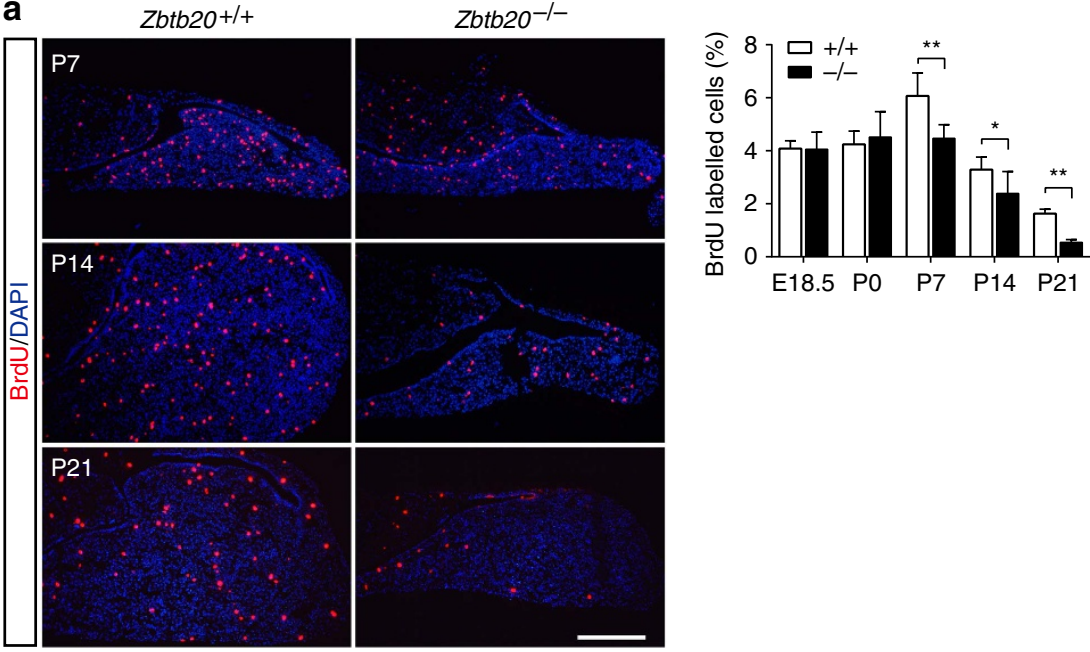

b

Zbtb20+/+

Zbtb20 ${ }^{-/-}$

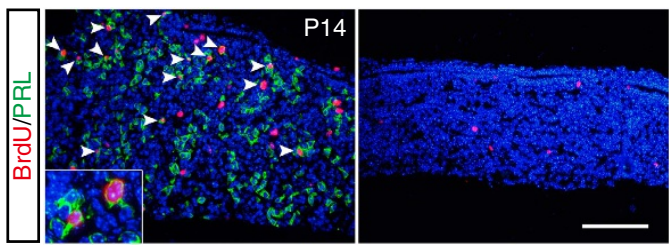

C

Zbtb20+/+

Zbtb20 ${ }^{-/-}$
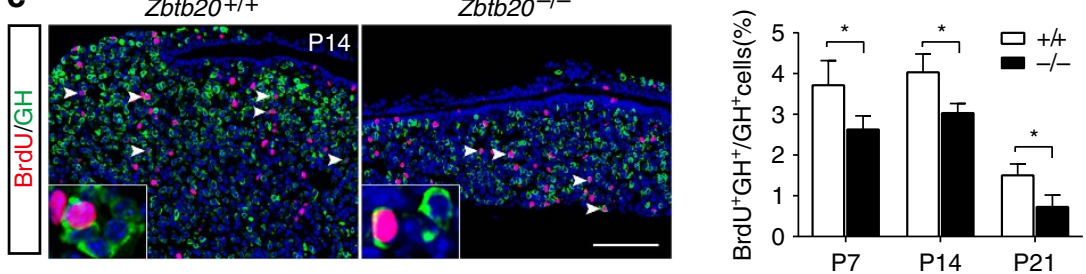

Figure 4 | Impaired postnatal cell proliferation in Zbtb20 ${ }^{-/-}$pituitary. (a) Immunohistochemical staining of BrdU was performed on E18.5 to P21

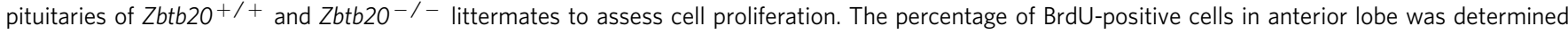
from counting serial sections of each pituitary. E18.5: $n=20$ sections from four animals; P0: $n=26$ sections from seven animals; P7: $n=31$ sections from five animals; P14: $n=34$ sections from five animals; and P21: $n=27$ sections from four animals. Values represent mean \pm s.e.m. ${ }^{\star} P<0.05$, ${ }^{\star \star} P<0.01$ (Student's t-test). (b,c) Immunohistochemical double staining of BrdU (red) and PRL (b) or GH (c) (green) was performed on the pituitary at the indicated age (P7 to P21). Arrowheads indicate double-positive cells, and inserts show the enlargement of double-positive cells. The percentage of double-positive cells among the GH-positive cells was determined by counting serial sections of each pituitary. P7: $n=8$ sections from three animals; P14: $n=12$ sections from three animals; and P21: $n=9$ sections from three animals. Values represent mean \pm s.e.m. ${ }^{\star} P<0.05$ (Student's $t$-test). Scale bar, (a) $200 \mu$ m; $(\mathbf{b}, \mathbf{c}) 100 \mu \mathrm{m}$.

levels in the pituitary at P14 (Fig. 6a,b). To determine whether ZBTB20 disruption resulted in a decrease in Pit-1 expression in somatotropes per se, we further performed double immunostaining of Pit-1 and GH. There was no significant difference of Pit-1 staining intensity in somatotropes between the two genotypes at P7 or P14 (Fig. 6c), which suggests that decreased Pit-1 expression in mutant pituitary most likely reflects the loss of Pit-1-expressing lactotropes and somatotropes. On the other hand, Pit-1 and PRL double staining showed that Pit-1 expression in PRL-positive cells was comparable between control and mutant pituitary at birth (Fig. 6d), suggesting that the defect of lactotrope specification in the absence of ZBTB20 was not likely due to the reduction of Pit-1 expression per se.

ZBTB20 expression in pituitary and hypothalamus. To understand the mechanisms by which ZBTB20 regulates anterior pituitary development and lactotrope specification, we examined its expression pattern in the developing pituitary and hypothalamus. The expression of ZBTB20 protein was first detected in hypothalamus as early as E13.5, but was excluded in pituitary at this time point (Supplementary Fig. 9). At E14.5, ZBTB20 protein was first detected in some cells of anterior pituitary, but absent in intermediate lobe (Fig. 7a). The number of ZBTB20-positive cells and intensity of ZBTB20 expression rapidly increased in the following 2 days. At E16.5, ZBTB20-positive cells were abundantly distributed over the entire anterior lobe, the expression levels of which were variable and much lower than hypothalamus. At P21, ZBTB20 expression was maintained at high levels in the pituitary (Fig. 7a). Double immunostaining of ZBTB20 and the pituitary hormones revealed that ZBTB20 was expressed by all the five endocrine cell types in anterior pituitary at P21 (Fig. 7b). Of note, most PRL-positive cells consistently expressed high levels of ZBTB20 from P0 to P14 (Fig. 7c). Furthermore, triple immunostaining with anti-GH, anti-PRL, and anti-ZBTB20 antibodies revealed high expression of ZBTB20 in somatolactotropes at both E18.5 and P4 (Fig. 7d). These data suggest that ZBTB20 might regulate anterior pituitary 
a

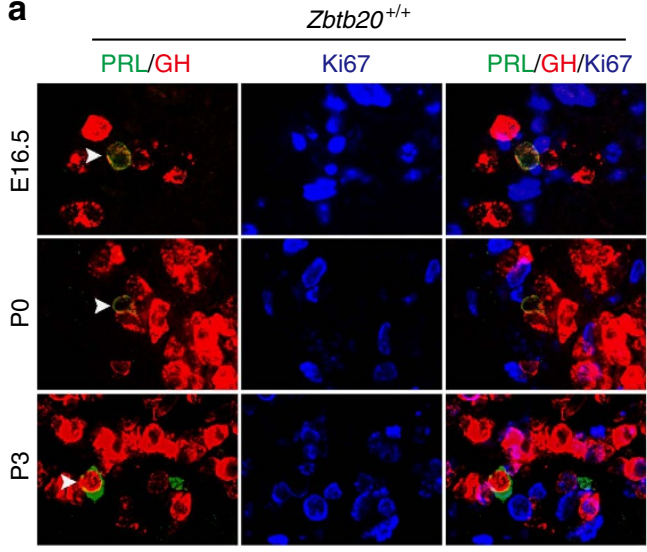

b

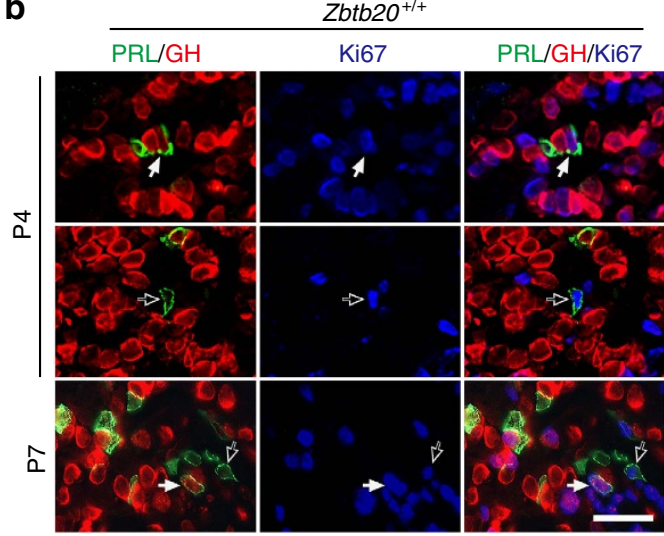

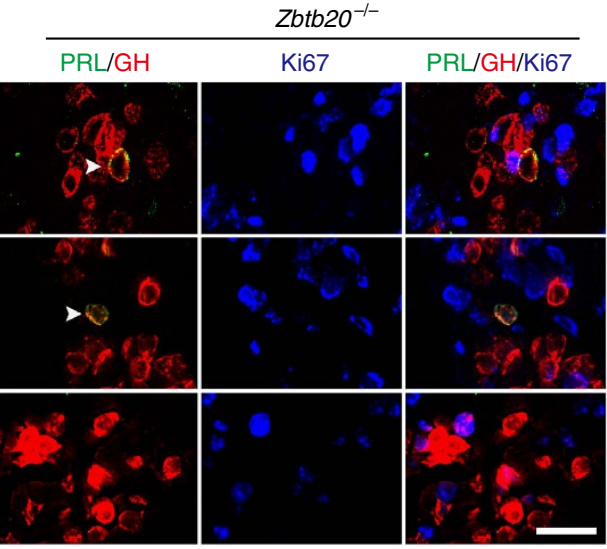

C

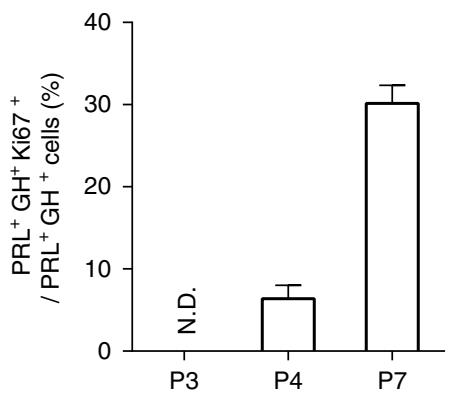

Figure 5 | Characterization of the proliferation of somatolactotropes from control and Zbtb20-null mice. (a,b) Triple immunostaining of GH (red), $\mathrm{PRL}$ (green) and $\mathrm{Ki} 67$ (blue) was performed on the pituitaries from control and mutant mice at the indicated age. Arrowheads (a) indicate $\mathrm{PRL}^{+} \mathrm{GH}^{+}$ double-positive cells, which are Ki67 negative. Solid white arrows (b) indicate PRL ${ }^{+} \mathrm{GH}^{+}$double-positive cells expressing Ki67, and open arrows (b) indicate PRL ${ }^{+} \mathrm{GH}^{-}$cells expressing Ki67. Scale bar, $25 \mu \mathrm{m}$. (c) The percentage of Ki67-expressing PRL ${ }^{+} \mathrm{GH}^{+}$cells in $\mathrm{PRL}^{+} \mathrm{GH}^{+}$cells was determined by counting serial sections of each pituitary at the indicated age. P3: $n=22$ sections from five animals; P4: $n=19$ sections from four animals; and P7: $n=18$ sections from four animals. Values represent mean \pm s.e.m. ND, not detectable.

development and lactotrope specification in cell-autonomous and/or nonautonomous manners.

Neuronal ZBTB20 is dispensable for lactotrope specification. Considering the role of hypothalamic dopamine in the negative regulation of lactotrope proliferation via its receptor Drd2, we first analysed the expression of the genes critically involved in dopamine synthesis and transportation. The mRNA expression levels of Th and Dat, which encode tyrosine hydroxylase and dopamine transporter, respectively, were comparable between control and mutant hypothalamus at P14 (Supplementary Fig. 10), implying that ZBTB20 deficiency might not alter the release of dopamine from hypothalamus to pituitary. To further determine whether hypothalamic ZBTB20 is involved in lactotrope specification, we inactivated this gene specifically in nervous system using Cre/loxP recombination system. The Nestin-Cre mouse line can mediate efficient gene deletion by E11 (ref. 32), a time point much earlier than the onset of ZBTB20 protein in nervous system including hypothalamus. Therefore we generated the pan-neuron-specific knockout mice of ZBTB20 (NS-ZB20KO) by crossing Zbtb20 loxP mice onto Nestin-Cre mice $^{33}$. Considering that this Cre line alone has some hypopituitarism in adulthood ${ }^{34}$, we analysed pituitary development of NS-ZB20KO mice only at early postnatal stage. Immunostaining analysis revealed that ZBTB20 expression was not affected in NS-ZB20KO pituitary, while totally abolished in the hypothalamus (Supplementary Fig. 11a). At P21, NS-ZB20KO pituitaries were smaller in size than control mice bearing Cre gene (Supplementary Fig. 11b). This raised the possibility that hypothalamic ZBTB20 could have a role in regulating pituitary development. However, there were no significant differences of either hormone mRNA levels or hormone-positive cell distribution between control and NS-ZB20KO mice (Supplementary Fig. $11 \mathrm{c}, \mathrm{d}$ ). These data at least indicate that neuronal ZBTB20 is dispensable for lactotrope specification. Thus, we reason that impaired lactotrope development in the absence of ZBTB20 is most likely due to an intrinsic defect of anterior pituitary.

Regulation of PRL expression by ZBTB20 in lactotropes. To explore the mechanisms by which ZBTB20 promotes lactotrope differentiation in a cell-autonomous fashion, we took advantage of a rat somatolactotrope cell line $\mathrm{GH} 3$ expressing both $\mathrm{GH}$ and PRL, and a rat lactotrope cell line MMQ, which expresses PRL but not GH. At both mRNA and protein levels, MMQ cells expressed much higher levels of $\mathrm{Prl}$ and Zbtb20 compared with GH3 cells (Supplementary Fig. 12). Considering that it is necessary for lactotrope differentiation to maintain PRL expression meanwhile extinguishing GH expression, we first examined the potential effect of ZBTB20 overexpression on Prl expression and lactotropic differentiation of $\mathrm{GH} 3$ cells. After $72 \mathrm{~h}$ of infection with recombinant replication-deficient adenoviruses co-expressing ZBTB20 and EGFP (hereafter Ad-ZBTB20) at the MOI of 400, 
a

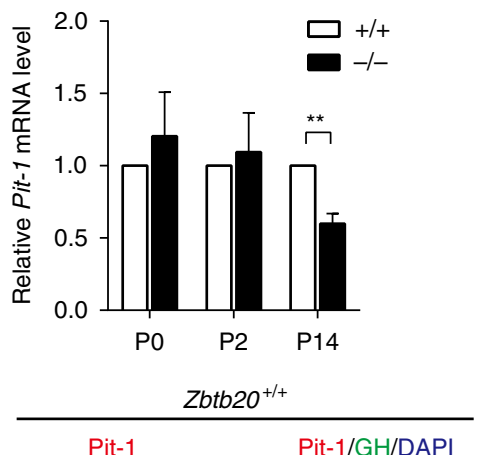

C
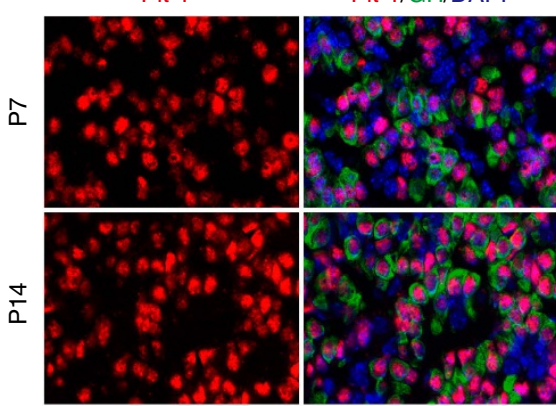

d

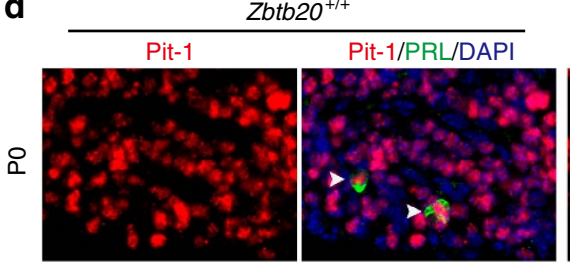

b
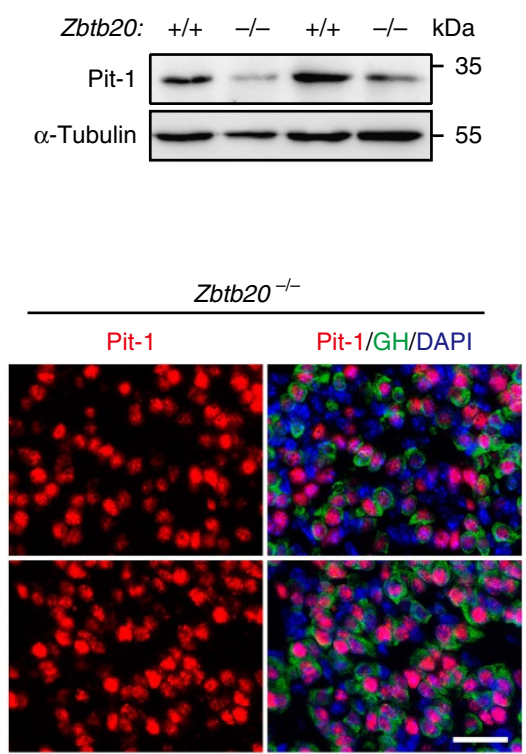

Zbtb20 ${ }^{-1-}$

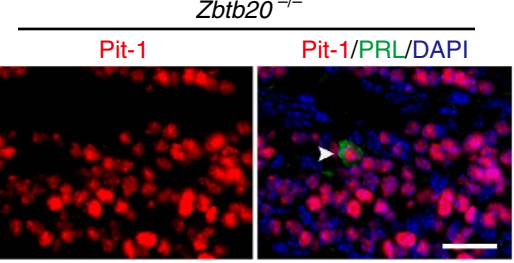

Figure 6 | Analysis of Pit-1 expression in anterior pituitary. (a) Quantification of Pit1 mRNA level at the indicated stages in $Z b t b 20^{+/+}$and $Z b t b 20^{-/-}$ mice by real-time RT-PCR. Results are presented as fold induction relative to mRNA levels in wild-type mice. A sample was pooled from four adenohypophyses of the same phenotype, and the experiment was repeated two times. Values represent mean \pm s.e.m. ${ }^{\star \star} P<0.01$ (Student's $t$-test). (b) Western blot of Pit1 proteins in pituitary extracts from 14-day-old $Z$ btb20 ${ }^{+/+}$and $Z$ btb20 ${ }^{-/-}$mice. A sample was pooled from five to eight adenohypophyses of the same phenotype, and the experiment was repeated twice. (c,d) Immunohistochemical double staining of Pit1 (red) and GH or PRL (green) is performed at the indicated stages in $\mathrm{Zbtb20}^{+/+}$and $\mathrm{Zbtb20}^{-/-}$pituitaries. Cell nuclei are stained with DAPI (blue). Arrowheads indicate PRL-positive cells. Scale bar, $25 \mu \mathrm{m}$.

more than $85 \%$ of $\mathrm{GH} 3$ cells were green fluorescent proteinpositive by flow cytometry assay (Supplementary Fig. 13). Compared with EGFP mock control, ZBTB20 overexpression in GH3 cells led to a marked increase in $\mathrm{Prl}$ expression and protein secretion into culture supernatants, but had no effect on $\mathrm{GH}$ expression at either mRNA or protein levels (Fig. 8a-c), suggesting that ZBTB20 upregulation alone is not sufficient to drive lactotropic differentiation from somatolactotropes. In addition, the expression of Pit1 or ER $\alpha$ was not affected by ZBTB20 overexpression in GH3 cells.

We next evaluated the effect of ZBTB20 knockdown on PRL expression of these two cell lines. Small interfering RNA (siRNA) against $Z$ btb20 was introduced into GH3 or MMQ cells by electroporation, which caused robust downregulation of ZBTB20 protein levels at day 3 post-treatment compared with control siRNA (Fig. 8d). Expectedly, ZBTB20 knockdown did not significantly affect the mRNA expression levels of PRL, GH or Pit-1 in GH3 cells (Fig. 8e), which is in agreement with the normal emergence of somatolactotropes in ZBTB20-null pituitary. In contrast, MMQ cells displayed a substantial decrease in $\mathrm{Prl}$ mRNA levels as well as its protein secretion in the culture supernatants as a result of ZBTB20 knockdown (Fig. 8e,f), whereas Pit-1 expression was not significantly affected, and $G H$ mRNA still undetectable. In addition, the cell growth rate of
MMQ was not significantly altered by ZBTB20 knockdown. Consistently, ChIP analyses revealed the binding of endogenous ZBTB20 protein to $\mathrm{Prl}$ promoter in MMQ but not GH3 cells (Fig. 8g). Therefore, these data suggested that ZBTB20 is specifically required to maintain $\mathrm{Prl}$ gene expression in lactotropes rather than somatolactotropes, but dispensable for $\mathrm{GH}$ gene repression in lactotropes.

We further performed $\mathrm{Prl}$ reporter assay to determine whether ZBTB20 regulates the transcriptional activity of $\mathrm{Prl}$ promoter. ZBTB20 knockdown in MMQ cells led to a robust decrease in the rat $\mathrm{Prl}$ promoter activity (Fig. $8 \mathrm{~h}$ ). On the other hand, the reporters harbouring different length of rat Prl promoters were not active in human embryonic kidney cell line 293T due to the absence of Pit-1 protein. Overexpression of Pit-1 alone could robustly activate the $\mathrm{Prl}$ reporters. More interestingly, ZBTB20 overexpression resulted in a marked increase in the transcriptional activity of the $\mathrm{Prl}$ promoters in $293 \mathrm{~T}$ cells in the presence of Pit-1 (Fig. 8i). These data strongly indicate that ZBTB20 regulates $\mathrm{Prl}$ gene transcription in a cell-autonomous manner.

Put together, we postulate a possible model for the regulation of lactotrope development by ZBTB20 (Supplementary Fig. 14). In this model, somatolactotropes are derived from Pit-1 lineage independently of ZBTB20, and undergo cell differentiation and proliferation before being specified into lactotropes in a 
a

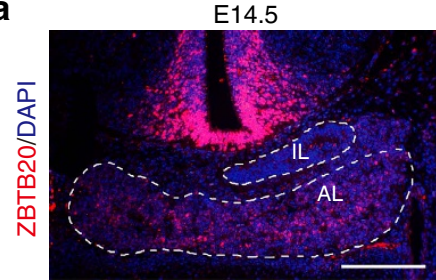

b
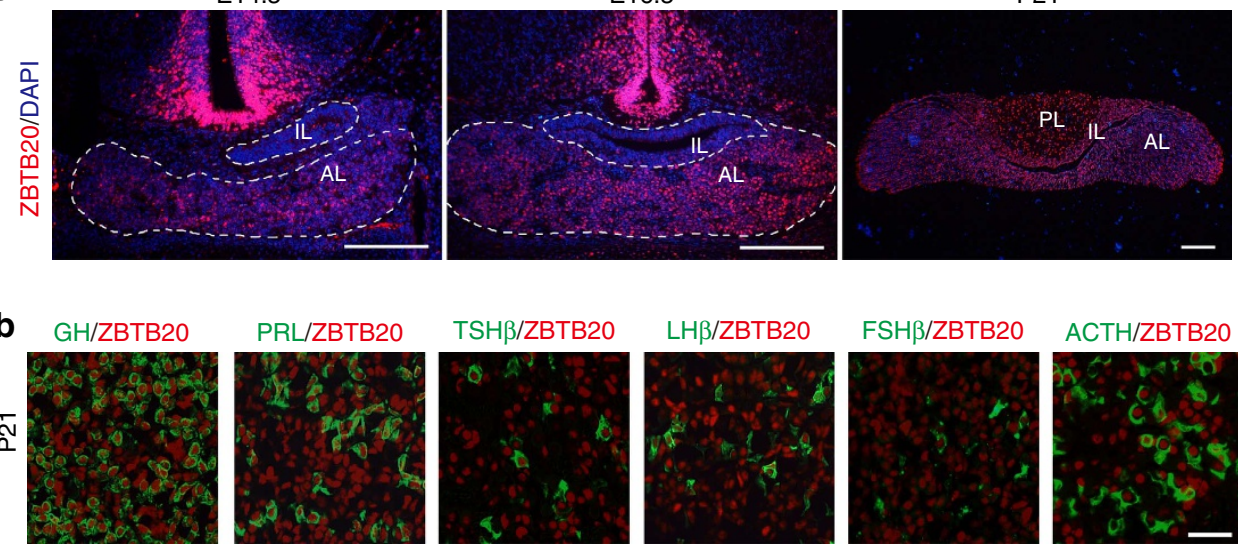

C

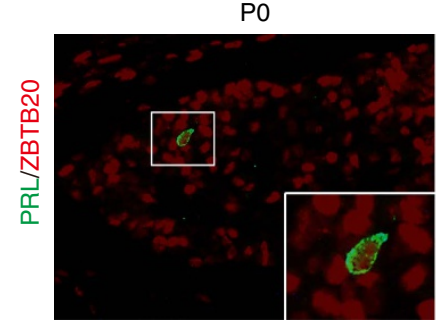

d

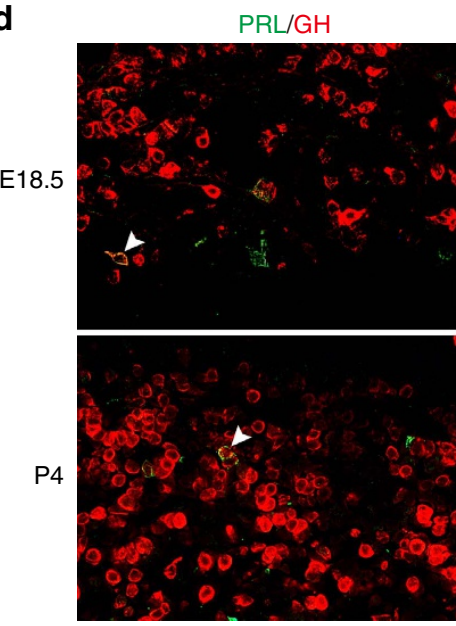

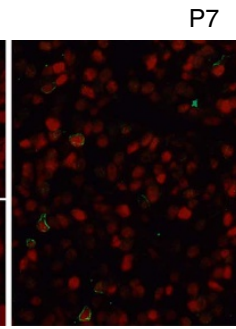

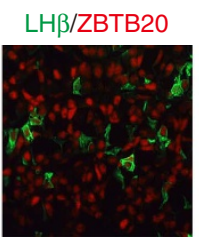

P7

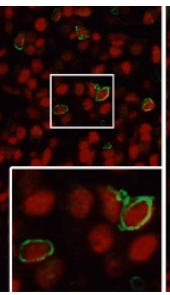

ZBTB20

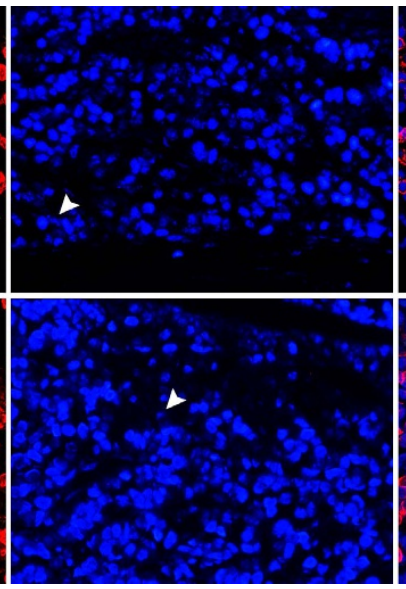

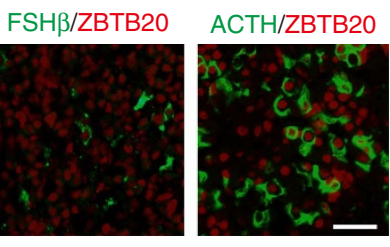

P14

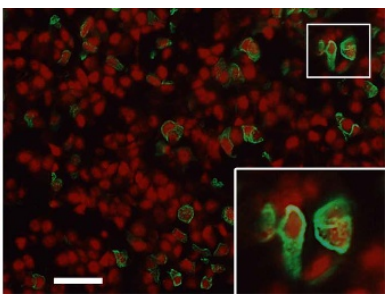

PRL/GH/ZBTB20

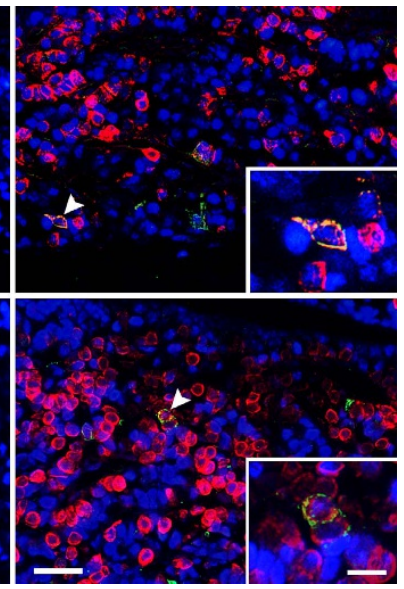

Figure 7 | Expression of ZBTB20 in the developing hypothalamus and pituitary. (a) ZBTB20 expression was detected by immunohistochemical staining at the indicated age on mouse adenohypophysis. White outline indicates adenohypophysis in embryo. AL, anterior lobe; IL, intermediate lobe; $\mathrm{PL}$, posterior lobe. Scale bar, $200 \mu \mathrm{m}$. (b,c) Immunohistochemical double staining of ZBTB2O (red) and hormones (green) in pituitary at the indicated age. Inserts are enlargement of the framed areas. Scale bar, $30 \mu \mathrm{m}$ (outside the inserts); $10 \mu \mathrm{m}$ (inside the inserts). (d) Triple staining of ZBTB20 (blue), PRL (green) and GH (red) in the pituitary at the indicated age. Arrowheads indicate PRL and GH double-positive cells expressing ZBTB20. Inserts show higher magnification of the area indicated by the arrowhead. Scale bar, $30 \mu \mathrm{m}$ (outside the inserts); $10 \mu \mathrm{m}$ (inside the inserts).

ZBTB20-dependent manner. Alternatively, lactotropes can be specified from somatolactotropes before the latter undergo cell replication, or even directly derived from GH-negative Pit-1 lineage, for either of which ZBTB20 is essential. Therefore, disruption of ZBTB20 leads to complete blockage of lactotrope specification and lineage expansion.

\section{Discussion}

In the present study, we for the first time establish that ZBTB20 is a lineage-specific regulator for anterior pituitary development. Global disruption of ZBTB20 leads to a decrease in somatotrope population and a total loss of mature lactotropes, with the manifestations of anterior pituitary hypoplasia, hypopituitary dwarfism and impaired mammary gland development, while other lineages (for example, thyrotropes, corticotropes and gonadotropes) are not significantly affected. Of note, both lactotropes and somatotropes are generated from Pit-1 lineage, however, their development is differentially regulated by ZBTB20. In the absence of ZBTB20, lactotrope development is completely blocked at the early stage of $\mathrm{PRL}^{+} \mathrm{GH}^{+}$somatolactotropic precursors as a result of the defect of cell specification, whereas somatotrope development is mildly impaired mainly because of the decrease in cell proliferation. Therefore, ZBTB20 plays critical roles in both lactotrope specification and somatotrope expansion in the developing anterior pituitary. 
a

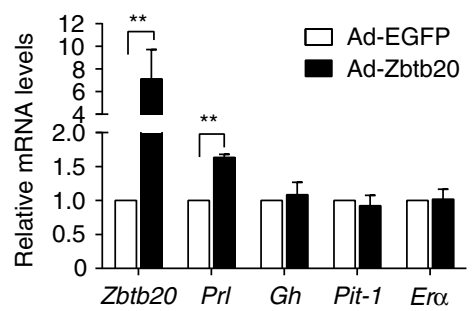

d

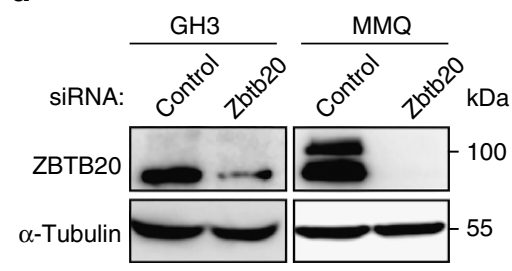

g

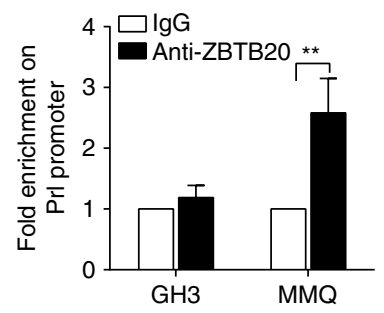

b

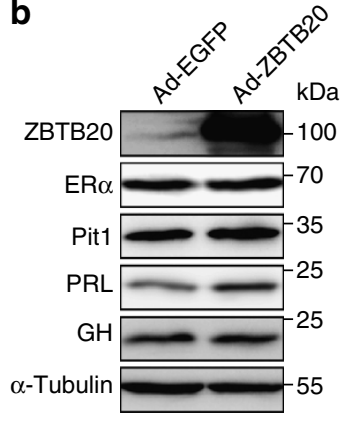

e $\quad \mathrm{GH} 3$

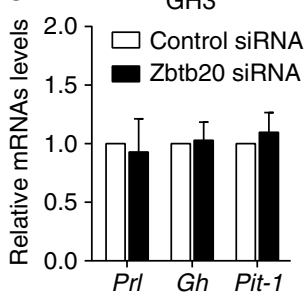

h

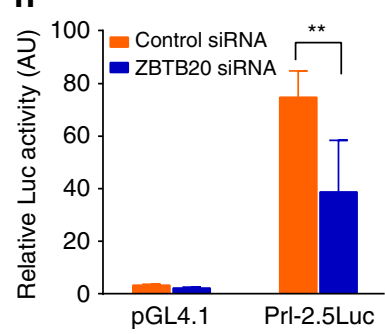

C
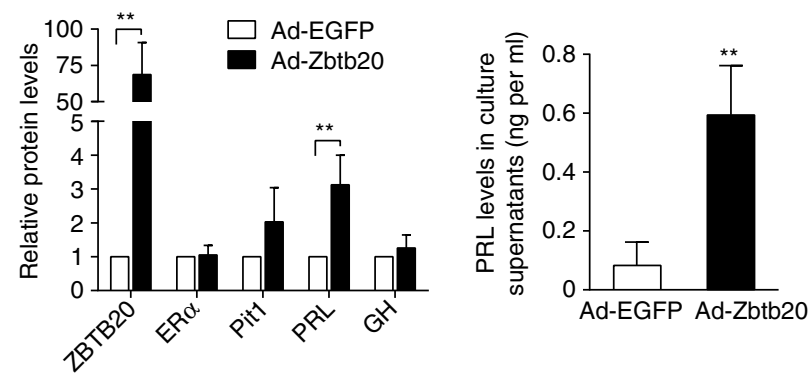

f

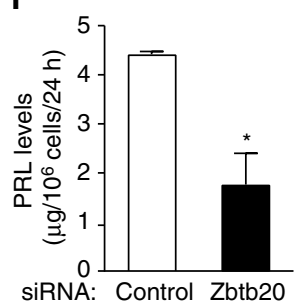

Figure 8 | ZBTB20 regulates Prl expression in a cell-autonomous fashion. (a-c) ZBTB20 overexpression in GH3 cells increases PRL expression and secretion without significant effect on GH expression. GH3 cells were infected with Ad-ZBTB20 or Ad-EGFP at MOI of 400 . Three days later, GH3 cells were subjected to gene expression analyses at mRNA levels by RT-PCR (a) and at protein levels by western blotting (b), with the expression levels presented as fold change relative to Ad-EGFP control, while PRL secretion was measured in the culture supernatants by ELISA (c). $n=4$. Values represent mean \pm s.e.m. ${ }^{\star \star} P<0.01$ versus Ad-EGFP (Student's $t$-test). (d-f) ZBTB20 knockdown decreases PRL expression and secretion in MMQ but not GH3 cells. Control or ZBTB2O siRNA was introduced into GH3 or MMQ cells by electroporation, respectively. Three days after transfection, the cells were harvested for western blotting (d) and quantitative RT-PCR analyses (e), and the culture supernatants were measured for PRL levels by ELISA (f). $n=4$. Values represent mean \pm s.e.m. ${ }^{\star} P<0.05$ versus control siRNA (Student's $t$-test). (g) ChIP assay showed the binding of ZBTB20 to rat Prl promoter in MMQ rather than $\mathrm{GH} 3$ cells. $n=3$. Values represent mean \pm s.e.m. ${ }^{\star \star} P<0.01$ versus control lgG (Student's $t$-test). (h) ZBTB20 knockdown decreases the transcriptional activity of $\mathrm{Prl}$ promoter in MMQ cells. MMQ cells were co-transfected with siRNA and expression plasmids by electroporation. Luciferase activity was measured at 48 -h post transfection, and normalized with internal control. $n=3$. Values represent mean \pm s.e.m. ${ }^{\star \star} P<0.01$ versus control siRNA (Student's t-test). (i) ZBTB20 overexpression enhances the transcriptional activity of the Prl promoters in 293T cells in the presence of Pit-1. Rat Prl promoters are $0.5,1.7 \mathrm{~kb}$, or $2.5 \mathrm{~kb}$ in length. $n=3$. Values represent mean \pm s.e.m. ${ }^{\star \star} P<0.01$ versus Pit-1 group (Student's $t$-test).

This study further excludes the possibility that hypothalamic ZBTB20 regulates lactotrope specification. The hypothalamus begins to express ZBTB20 earlier and at much higher levels than pituitary during embryonic development, raising the possibility that hypothalamic ZBTB20 may regulate pituitary development in an extrinsic manner. However, Nestin-Cre-mediated neuron-specific ablation of ZBTB20 did not affect pituitary development and lactotrope specification during early postnatal stage. Because this Cre transgenic line itself has mild hypopituitarism in adulthood ${ }^{34}$, it is not an ideal model to investigate the role of hypothalamic ZBTB20 in postnatal pituitary development. Currently, using this line, we at least conclude that hypothalamic ZBTB20 is dispensable for lactotrope specification. This conclusion is also supported by the observation that the expression levels of $T h$ and Dat were not altered in hypothalamus by the loss of Zbtb20, which are involved in dopamine synthesis and transportation. On the other hand, ZBTB20 is expressed by somatolactotropes in the developing anterior pituitary. Therefore, most likely, it is pituitary ZBTB20 that regulates lactotrope specification. To more precisely determine the role of hypothalamic ZBTB20 in pituitary development, other neuron-specific Cre lines, e.g. directed by endogenous Tau promoter ${ }^{35}$, may be a good option. On the other hand, knockdown of ZBTB20 in lactotrope cell line MMQ results in a robust decrease in PRL protein expression and secretion. Furthermore, ZBTB20 could bind to and regulate the transcriptional activity of $\mathrm{Prl}$ promoter in MMQ cells. These data strongly suggest that ZBTB20 most likely regulates lactotrope specification in a cell-autonomous manner.

The ontogeny of lactotropes is closely related with somatotropes. The early lactotropes also express $\mathrm{GH}$ in the rat and cow $^{11}$. Importantly, a majority of lactotropes are depleted in the transgenic mice using rat $\mathrm{GH}$ promoter to target diphtheric toxin A-mediated destruction of GH-expressing cells ${ }^{12}$. Similarly, both somatotropes and lactotropes are largely depleted in the GH promoter-driven thymidine kinase transgenic mice after consecutive administration of the synthetic nucleoside FIAU (1-(2-deoxy-2-fluoro- $\beta$ - $\delta$-arabinofranosyl)-5-iodouracil) to kill 
dividing cells during embroynic and postnatal development ${ }^{13}$. These observations strongly suggest that a majorty of lactotropes may arise from the dual hormone-expressing somatolactotropes. However, the molecular mechanism about lactotrope specification has not been defined. We found that ZBTB20 expression begins to emerge at E14.5 in pituitary. Of note, ZBTB20 is also expressed by somatolactotropes, and ZBTB20 deficiency leads to complete blockage of lactotrope specification at the stage of somatolactotropes. In ZBTB20-null mice, $\mathrm{PRL}^{+} \mathrm{GH}^{+}$precursors are consistently present between E15.5 to P0, but display a complete defect to differentiate into $\mathrm{PRL}^{+} \mathrm{GH}^{-}$cells, which occurs as early as at E18.5 in control mice. These data suggest that ZBTB20 is essential for lactotrope specification from GH-expressing precursors. Alternatively, lactotropes can also develop independently of somatotrope lineage ${ }^{12,36}$. However, as a result of ZBTB20 disruption, PRL single-positive cells were not detected in either embryonic or postnatal pituitary. Therefore, we reason that ZBTB20-regulated cell differentiation is critical for lactotrope specification in both somatolactotrope-dependent and -independent pathways.

Our study further points out that ZBTB20-regulated cell differentiation is critical for lactotrope lineage expansion. The previous transgenic study show that proliferating GH-positive cells are critical for postnatal lactotrope development ${ }^{13}$; however, the dynamics of somatolactotrope proliferation has not been characterized so far. Our data demonstrate that differentiating somatolactotropes are not replicating until P4. From P7 to P14, somatolactotropes undergo robust cell proliferation, which to great extent accounts for the lactotrope lineage expansion. Therefore, lactotrope specification can occur before or after somatolactotrope replication. In addition, specified lactotropes are also highly proliferating at P14, which may also contribute to lactotrope lineage expansion. ZBTB20 deletion leads to a complete blockage of lactotrope lineage expansion due to the failure of somatolactotropes to enter the cell replication window and lineage specification. The fate of ZBTB20-null somatolactotropes could be apoptosis. However, due to the scarcity of the cell population in the perinatal pituitary, it is hard to trace somatolactotropic apoptosis. Alternatively, they could be differentiated to other lineages than lactotropes, for example somatotropes. To confirm this possibility, lineage tracing experiment needs to be performed in the future.

Pit-1 is crucial for the development of somatotropes, lactotropes and thyrotropes. Pit-1 mutation causes the combined deficiency of the three lineages. Particularly, in response to oestrogen, ER $\alpha$ promotes PRL expression by synergistic interaction with Pit-1, and largely accounts for the postnatal expansion of lactotropes ${ }^{7,37,38}$. However, ablation of ER $\alpha$ has no effects on lactotrope specification despite of a decrease in PRL expression levels and lactotrope cell number ${ }^{16}$. We found that Pit-1 expression was not significantly altered in ZBTB20-null lactotropic progenitors compared to control at $\mathrm{P} 0$, raising a possibility that ZBTB20 may regulate lactotrope specification through interaction with Pit-1. Our reporter assay revealed that ZBTB20 could enhance the transcriptional activity of $\mathrm{Prl}$ promoter in the presence of Pit-1, suggesting that ZBTB20 may be critically involved in the activation of $P r l$ gene by Pit-1 probably as a coactivator. In the previous studies, we have established ZBTB20 as a transcriptional repressor of the genes encoding alpha-fetoprotein (AFP) in liver ${ }^{23,39}$, fructose-1, 6,-bisphosphotase 1 (Fbp1) in pancreatic $\beta$ cells ${ }^{26}$, I $\mathrm{KB} \alpha$ in macrophages $^{40}$, and Sox9 in hypertrophic chondrocytes ${ }^{41}$. Put together, we thus postulate that the transcriptional regulatory functions of ZBTB20 could be context-dependent. Finally, it is also possible that ZBTB20 regulates the expression of other unidentified targets than $\mathrm{Prl}$ gene, which are critical for lactotrope development. Therefore, the exact molecular mechanism by which ZBTB20 regulates pituitary development and lactotrope specification needs further investigation in the future.

In summary, ZBTB20 is required for anterior pituitary development and lactotrope specification. Our identification of ZBTB20 as a crucial determinant of lactotrope specification provides insight into the ontogeny of lactotropes, which will help to unravel the cellular and molecular basis for pituitary deficiency.

\section{Methods}

Animals. ZBTB20-null mice were generated by crossing between $Z$ btb20 $+1-$ mice ${ }^{24}$, and central nervous system-specific Zbtb20 knockout mice were generated by repeated crossing $Z b t b 20^{f l o x}$ mice with Nestin-cre transgenic mice 23,33 . Mice were bred in specific pathogen-free conditions. The mice used for the experiments were homozygous mutant mice and their wild-type littermates, and backcrossed into the C57BL/6 background for a minimum of 10 generations. Because a vast majority of homozygous $Z b t b 20^{-1-}$ mice die within one month of age, experiments were performed in mice within 3 weeks of age. All experiments were performed in accordance with the guidelines of the Second Military Medical University Animal Ethics Committee.

Tissue preparation. The morning when vaginal plug was observed was designated as embryonic day 0.5 (E0.5), and the day of birth was P0. For morphological analysis, embryos were collected from timed-pregnant females, and their heads were fixed with $4 \%$ paraformaldehyde overnight and embedded in paraffin. Mouse pups were anaesthetized by hypothermia ( $\mathrm{P} 0-\mathrm{P} 4)$ or $5 \%$ urethane $(30 \mu \mathrm{l}$ per $\mathrm{g}$ of body weight; P7-P28), and perfused transcardially with $4 \%$ paraformaldehyde. Pituitary glands were dissected out by stereo microscope, immersed in $4 \%$ paraformaldehyde for $40 \mathrm{~min}$ to $2 \mathrm{~h}$, and embedded in paraffin. Consecutive serial sections $(4 \mu \mathrm{m})$ at $100-\mu \mathrm{m}$ intervals were collected for immunostaining.

Cell proliferation and apoptosis assays. For BrdU experiments, timed-pregnant mice and postnatal $0,7,14$ and 21 days old mice were injected intraperitoneally with $\mathrm{BrdU}$ (100 mg per kg of body weight) and killed $2 \mathrm{~h}$ later for immunostaining analysis using anti-BrdU antibody. For apoptosis assays, serial coronal pituitary sections were subjected to TUNEL staining following the manufacturer's protocol (Promega).

Histology and immunohistochemistry. H\&E staining of pituitary and whole-mount analysis of mammary gland were performed according to standard protocols ${ }^{42}$. Pituitary or hypothalamus sections were deparaffinized, rehydrated, blocked in PBS with bovine serum albumin and incubated with primary antibody. The following primary antibodies were obtained from Dr A.F.Parlow at the National Institutes of Health of USA to identify pituitary cell lineages: rabbit antiGH, -PRL, -TSH $\beta$, -FSH $\beta$ and -ACTH antibodies. For single immunofluorescent staining, tissue sections were incubated overnight at $4{ }^{\circ} \mathrm{C}$ with the above hormone antibodies (1:2,000), anti-LH $\beta$ antibody (1:2,000, Acris), anti-ZBTB20 antibody 9A10 (home-made, 1:2,000), anti-Drd2 antibody (1:1,000, Millipore), anti-Sox2 antibody (1:2,000, Santa Cruz), anti-BrdU antibody (1:2,000, Sigma-Aldrich), and anti-Pit1 antibody (1:500, Abcam). The signals were amplified by TSA Plus DNP system (thereafter TSA system, NEN Life Science Products Inc., Boston) ${ }^{25}$, and visualized with Alexa Fluor 594. For hormones and ZBTB20 or BrdU double labelling, pituitary sections were incubated with different hormone antibodies $(1: 1,000$, NHPP) and anti-ZBTB20 antibody 9 A10 $(1: 1,000)$ or anti-BrdU antibody $(1: 2,000)$, signals were amplified by TSA system, and visualized with Alexa Fluor 594 and 488, respectively. For PRL and GH colabelling, pituitary sections were incubated with goat anti-PRL antibody (1:100, Santa Cruz) and rabbit anti-GH antibody (1:100, NHPP), followed by incubation with Alexa Fluor 594- or Alexa Fluor 488-conjugated secondary antibodies (1:200, Molecular Probes, Life Technologies), respectively. For Pit-1 and PRL or GH double labelling, pituitary sections were incubated with mouse anti-Pitl antibody (1:250, Abcam) and rabbit anti-PRL or -GH antibodies (1:100, NHPP). Pit-1 signal was amplified by TSA system and visualized with Alexa Fluor 594, while PRL and GH signals were visualized by incubation with Alexa Fluor 488-conjugated secondary antibodies. For triple staining of PRL/GH/Ki67 or PRL/GH/ZBTB20, pituitary sections were incubated with goat anti-PRL antibody (1:100, Santa Cruz), rabbit anti-GH antibody (1:100, NHPP) and rat anti-Ki67 antibody (1:1,000, Dako) or mouse anti-ZBTB20 antibody 9A10 (1:1,000). Ki67 and ZBTB20 signals were amplified by TSA system and visualized with Alexa Fluor 350, while PRL and GH signals were visualized by incubation with Alexa Fluor 488- and Alexa Fluor 594-conjugated secondary antibodies, respectively.

In situ hybridization. After perfused with $4 \%$ paraformaldehyde in PBS, pituitary glands of mice were fixed with $4 \%$ paraformaldehyde for $2 \mathrm{~h}$ and saturated with $20 \%$ sucrose in PBS overnight at $4{ }^{\circ} \mathrm{C}$. Pituitary were embedded in optimal cutting 
temperature (OCT) compound and sectioned at $10 \mu \mathrm{m}$. Antisense riboprobe of Pit-1 labelled with digoxygenin-UTP were transcribed from complementary DNA (cDNA) clone. After overnight hybridization with riboprobe, coronal pituitary sections were detected with anti-digoxygenin (Roche) antibody conjugated to alkaline phosphatase, and developed by nitro blue tetrazolium.

Quantitative RT-PCR. Adenohypophyses were isolated under stereo microscope and pooled per genotype. Total RNA was extracted from adenohypophyses, livers, and hypothalamus using TRIzol reagent (Invitrogen, Life Technologies), and treated with DNase I (Fermentas, Thermo). cDNAs were produced with a ReverTra Ace-a Kit (Toyobo), and real-time PCR was performed with a QuantiFast SYBR Green PCR Kit (Qiagen). Primer sequences are listed in Supplementary Table 1. Data were normalized to Gapdh expression and expressed as the fold increase of control.

Western blotting. Adenohypophyses pooled per genotype were lysed in RIPA buffer supplemented with protease inhibitor mixture. Protein concentrations of the extracts were measured with BCA protein assay kit (Picerce, Thermo). Proteins were resolved on $4-12 \%$ gradient SDS/PAGE, transferred to PVDF membranes, and immunoblotted with anti-ZBTB20 clone 9A10 antibody (1:2,000), anti-PRL antibody (1:1,000, NHPP), anti-GH antibody (1:1,000, NHPP), anti-POMC antibody (1:500, NHPP), anti-Pit1 antibody (1:500, Abcam), anti-DRD2 antibody (1:500, Millipore), and anti- $\alpha$-Tubulin antibody (1:5,000, Proteintech). Secondary antibodies (1:10,000, Vector) coupled to horseradish peroxidase were used, and blots were developed with a West Pico enhanced chemiluminescence kit (Pierce, Thermo). Uncropped scans are shown in Supplementary Fig. 15.

ELISA for IGF-1 and pituitary hormones. For IGF-1 measurement, blood was collected by retro-orbital puncture under $5 \%$ urethane anaesthesia after 6 -h fasting. Plasma IGF-1 levels were measured by using a mouse IGF-1 ELISA kit (Alpco, Salem, NH). For pituitary hormones measurement, a single adenohypophysis was lysed in $100 \mu \mathrm{l}$ RIPA buffer and diluted by PBS to $1 \mathrm{ml}$. Anterior pituitary hormones were measured with corresponding mouse ELISA kits (Alpco).

Cell counts. To quantify proliferating cells, BrdU-positive cells were counted randomly in a minimum of 600 anterior pituitary cells from the coronal sections. Three to seven sections were selected from each pituitary; and four to seven animals were used for each time point. The total number of positive cells was expressed as the percentage with the same area. To quantify $\mathrm{PRL}^{+} \mathrm{GH}^{-}$cells, $\mathrm{PRL}^{+} \mathrm{GH}^{+}$cells and proliferating PRL ${ }^{+} \mathrm{GH}^{+}$cells, all PRL-positive cells, $\mathrm{PRL}^{+}$ $\mathrm{GH}^{+}$cells and triple positive cells on coronal sections were counted. Two to six sections were selected from each animal, and two to five animals were used for each time point.

Cell culture and adenoviral infection. Rat GH3 and MMQ cells were obtained from American Type Culture Collection (ATCC), and cultured in F-12K Nutrient Mixture (Gibco, Life Technologies) supplemented with 15\% horse serum, $2.5 \%$ fetal bovine serum, and $1 \%$ penicillin/streptomycin. Recombinant adenoviral vector expressing ZBTB20 was constructed by inserting human ZBTB20 cDNA into pAd-track (Stratagene) under the control of CMV promoter, and in vitro recombination with adenoviral backbone vector pAdEasy-1 and subsequent viral packaging in 293A cells were performed according to the instructions of the manufacturer. Recombinant adenoviruses were purified by $\mathrm{CsCl}$ ultracentrifugation, and subjected to dialysis against PBS before titration in 293A cells. GH3 cells were infected with recombinant adenoviruses co-expressing ZBTB20 and EGFP (Ad-ZBTB20) or mock adenoviruses expressing EGFP (Ad-EGFP) at MOI of 400, and incubated for 3 days for the expression of ZBTB20 before harvesting for analysis. The efficiency of infection was assessed by measurement of percentage of EGFP-positive cells using a flow cytometry (Becton Dickinson).

RNA interference analysis. After wash with PBS twice, $5 \times 10^{5}$ GH3 or MMQ cells were resuspended in BTXpress High Performance Electroporation solution (Harvard Apparatus, MA, USA) containing $2.5 \mu \mathrm{mol}$ of control or Zbtb20 siRNA (Dhmarcon, PA, USA), and subjected to electroporation in a 2-mm cuvette using a BTX ECM830 electroporator ${ }^{41}$, with the setting of one pulse of 170 volts for $15 \mathrm{~ms}$. Then cells were plated in normal culture media. Twenty-four hours after transfection, the cells were collected by centrifugation, and resuspended in fresh media with the density of $1 \times 10^{5} \mathrm{ml}^{-1}$. After culture for another $48 \mathrm{~h}$ at $37^{\circ} \mathrm{C}$, the culture supernatants were collected for PRL measure with ELISA kit (Alpco), and the cells were subjected to mRNA and protein expression analyses.

Chromatin immunoprecipitation analysis. Fragmented chromatin from GH3 or MMQ cells was incubated overnight with anti-ZBTB20 antibody 9A10 (homemade, $0.5 \mu \mathrm{g}$ per $10^{6}$ cells), anti-acetylated histone 3 (Millipore, $0.5 \mu \mathrm{g}$ per $10^{6}$ cells) as positive control, or isotype IgG (Upstate, $0.5 \mu \mathrm{g}$ per $10^{6}$ cells) as negative control, and followed by incubation with Dynabead-conjugated Protein G (Invitrogen). Purified chromatin DNA was subjected to real-time PCR analysis with the primers for rat $\mathrm{Prl}$ promoter, the sequence of which is $5^{\prime}$-TTTGGGGTCAGAAGAGGC- $3^{\prime}$ and $5^{\prime}$-TTGTGGAAGGAGCGCAGT- $3^{\prime}$. The data were analysed using the formula of $2^{-\Delta \Delta \mathrm{Ct}}$, where $\Delta \Delta \mathrm{CT}=(\mathrm{Ct}[\mathrm{IP}]-\mathrm{Ct}[\text { input }])_{\mathrm{SA}^{-}}(\mathrm{Ct}[\mathrm{IP}]-C \mathrm{t}[\text { input }])_{\mathrm{NS}}$ $\mathrm{SA}=$ Specific antibody, NS $=$ Non-specific antibody. Three independent ChIPs were performed.

Promoter luciferase reporter assay. A $\mathrm{Prl}$ promoter-driven firefly luciferase reporter (\#608) was first constructed by cloning rat $\mathrm{Prl}$ promoter region (covering from -2486 to +65 , named PRL-2.5Luc) into the pGL4.10 vector (Promega) at the sites of SacI and Bgl2, the sequence of which was confirmed by DNA sequencing. Truncated Prl reporters were obtained by the removal of $5^{\prime}$ end of $\mathrm{Prl}$ promoter from the reporter PRL-2.5Luc with restrictive digestion, thereby containing $\sim 1.7$ or $0.5 \mathrm{~kb}$ of $\mathrm{Prl}$ proximal promoters (Prl-1.7Luc and Prl-0.5Luc), respectively. MMQ cells were co-transfected with control or ZBTB20 siRNA $(2.5 \mu \mathrm{mol})$ plus $500 \mathrm{ng}$ of reporter plasmids by electroporation. HEK $293 \mathrm{~T}$ cells (ATCC) were co-transfected in 24-well plate with reporter constructs, ZBTB20 and/or Pit-1 expression plasmids using Effectene transfection reagent (Qiagen) The pGL4.1 empty vector served as a blank control. Renilla luciferase plasmid RL-TK was co-transfected as internal control to normalize the luciferase activity. Forty-eight hours after transfection, the cells were collected, and disrupted with passive lysis buffer (Promega). Luciferase activity was measure using a Dual-Luciferase Reporter Assay System (Promega) on a GloMAX luminometer (Promega), and firefly luciferase activity was normalized to renila luciferase activity of internal control. Experiments were performed in triplicate and repeated three times.

Statistical analysis. Quantitative data are shown as mean \pm s.e.m. Differences among more than two groups were performed by two-way analysis of variance, and differences between two groups were performed with Student $t$-test. $P$ values of $<0.05$ were considered statistically significant.

\section{References}

1. Zhu, X., Gleiberman, A. S. \& Rosenfeld, M. G. Molecular physiology of pituitary development: signaling and transcriptional networks. Physiol. Rev. 87, 933-963 (2007).

2. Kelberman, D., Rizzoti, K., Lovell-Badge, R., Robinson, I. C. \& Dattani, M. T. Genetic regulation of pituitary gland development in human and mouse. Endocr. Rev. 30, 790-829 (2009).

3. Moraes, D. C., Conceicao, F. L., Vaisman, M. \& Ortiga-Carvalho, T. M. Pituitary development: a complex, temporal, regulated process dependent on specific transcriptional factors. J. Endocrinol. (2012).

4. Suga, H. et al. Self-formation of functional adenohypophysis in threedimensional culture. Nature 480, 57-62 (2011).

5. Scully, K. M. et al. Allosteric effects of Pit-1 DNA sites on long-term repression in cell type specification. Science 290, 1127-1131 (2000).

6. Zhu, X. et al. Sustained Notch signaling in progenitors is required for sequential emergence of distinct cell lineages during organogenesis. Genes Dev. 20, 2739-2753 (2006).

7. Simmons, D. M. et al. Pituitary cell phenotypes involve cell-specific Pit-1 mRNA translation and synergistic interactions with other classes of transcription factors. Genes Dev. 4, 695-711 (1990).

8. Li, S. et al. Dwarf locus mutants lacking three pituitary cell types result from mutations in the POU-domain gene pit-1. Nature 347, 528-533 (1990).

9. Lin, C., Lin, S. C., Chang, C. P. \& Rosenfeld, M. G. Pit-1-dependent expression of the receptor for growth hormone releasing factor mediates pituitary cell growth. Nature 360, 765-768 (1992).

10. Tatsumi, K. et al. Cretinism with combined hormone deficiency caused by a mutation in the PIT1 gene. Nat. Genet. 1, 56-58 (1992).

11. Hoeffler, J. P., Boockfor, F. R. \& Frawley, L. S. Ontogeny of prolactin cells in neonatal rats: initial prolactin secretors also release growth hormone. Endocrinology 117, 187-195 (1985).

12. Behringer, R. R., Mathews, L. S., Palmiter, R. D. \& Brinster, R. L. Dwarf mice produced by genetic ablation of growth hormone-expressing cells. Genes Dev. 2 , 453-461 (1988).

13. Borrelli, E., Heyman, R. A., Arias, C., Sawchenko, P. E. \& Evans, R. M. Transgenic mice with inducible dwarfism. Nature 339, 538-541 (1989).

14. Ogasawara, K. et al. Hormonal regulation of prolactin cell development in the fetal pituitary gland of the mouse. Endocrinology 150, 1061-1068 (2009).

15. Stefaneanu, L., Powell-Braxton, L., Won, W., Chandrashekar, V. \& Bartke, A. Somatotroph and lactotroph changes in the adenohypophyses of mice with disrupted insulin-like growth factor I gene. Endocrinology 140, 3881-3889 (1999).

16. Scully, K. M. et al. Role of estrogen receptor-alpha in the anterior pituitary gland. Mol. Endocrinol. 11, 674-681 (1997).

17. Hikake, T., Hayashi, S., Iguchi, T. \& Sato, T. The role of IGF1 on the differentiation of prolactin secreting cells in the mouse anterior pituitary. J. Endocrinol. 203, 231-240 (2009). 
18. Lopez-Fernandez, J. et al. Differentiation of lactotrope precursor GHFT cells in response to fibroblast growth factor-2. J. Biol. Chem. 275, 21653-21660 (2000).

19. Zhou, M. et al. Fibroblast growth factor 2 control of vascular tone. Nat. Med. 4, 201-207 (1998)

20. Ortega, S., Ittmann, M., Tsang, S. H., Ehrlich, M. \& Basilico, C. Neuronal defects and delayed wound healing in mice lacking fibroblast growth factor 2 . Proc. Natl Acad. Sci. USA 95, 5672-5677 (1998).

21. Dono, R., Texido, G., Dussel, R., Ehmke, H. \& Zeller, R. Impaired cerebral cortex development and blood pressure regulation in FGF-2-deficient mice. EMBO J. 17, 4213-4225 (1998).

22. Zhang, W. et al. Identification and characterization of DPZF, a novel human $\mathrm{BTB} / \mathrm{POZ}$ zinc finger protein sharing homology to BCL-6. Biochem. Biophys. Res. Commun. 282, 1067-1073 (2001).

23. Xie, Z. et al. Zinc finger protein ZBTB20 is a key repressor of alpha-fetoprotein gene transcription in liver. Proc. Natl Acad. Sci. USA 105, 10859-10864 (2008).

24. Sutherland, A. P. et al. Zinc finger protein Zbtb20 is essential for postnatal survival and glucose homeostasis. Mol. Cell. Biol. 29, 2804-2815 (2009).

25. Xie, Z. et al. Zbtb20 is essential for the specification of CA1 field identity in the developing hippocampus. Proc. Natl Acad. Sci. USA 107, 6510-6515 (2010).

26. Zhang, Y. et al. The zinc finger protein ZBTB20 regulates transcription of fructose-1,6-bisphosphatase 1 and beta cell function in mice. Gastroenterology 142, 1571-1580 e1576 (2012).

27. Li, H., Zeitler, P. S., Valerius, M. T., Small, K. \& Potter, S. S. Gsh-1, an orphan Hox gene, is required for normal pituitary development. EMBO J. 15, 714-724 (1996).

28. Kelly, M. A. et al. Pituitary lactotroph hyperplasia and chronic hyperprolactinemia in dopamine D2 receptor-deficient mice. Neuron 19, 103-113 (1997)

29. Perez Millan, M. I. et al. Selective disruption of dopamine D2 receptors in pituitary lactotropes increases body weight and adiposity in female mice. Endocrinology 155, 829-839 (2014).

30. Fauquier, T., Rizzoti, K., Dattani, M., Lovell-Badge, R. \& Robinson, I. C. SOX2-expressing progenitor cells generate all of the major cell types in the adult mouse pituitary gland. Proc. Natl Acad. Sci. USA 105, 2907-2912 (2008).

31. Fu, Q. et al. The adult pituitary shows stem/progenitor cell activation in response to injury and is capable of regeneration. Endocrinology 153, 3224-3235 (2012)

32. Tronche, F. et al. Disruption of the glucocorticoid receptor gene in the nervous system results in reduced anxiety. Nat. Genet. 23, 99-103 (1999).

33. Ren, A. J. et al. ZBTB20 regulates nociception and pain sensation by modulating TRP channel expression in nociceptive sensory neurons. Nat. Commun. 5, 4984 (2014).

34. Galichet, C., Lovell-Badge, R. \& Rizzoti, K. Nestin-Cre mice are affected by hypopituitarism, which is not due to significant activity of the transgene in the pituitary gland. PLoS ONE 5, el1443 (2010).

35. Korets-Smith, E. et al. Cre recombinase specificity defined by the tau locus. Genesis 40, 131-138 (2004).

36. Luque, R. M. et al. Reporter expression, induced by a growth hormone promoter-driven Cre recombinase ( $\mathrm{rGHp}$-Cre) transgene, questions the developmental relationship between somatotropes and lactotropes in the adult mouse pituitary gland. Endocrinology 148, 1946-1953 (2007).
37. Day, R. N., Koike, S., Sakai, M., Muramatsu, M. \& Maurer, R. A. Both Pit-1 and the estrogen receptor are required for estrogen responsiveness of the rat prolactin gene. Mol. Endocrinol. 4, 1964-1971 (1990).

38. Nowakowski, B. E. \& Maurer, R. A. Multiple Pit-1-binding sites facilitate estrogen responsiveness of the prolactin gene. Mol. Endocrinol. 8, 1742-1749 (1994).

39. Zhang, H. et al. ZBTB20 is a sequence-specific transcriptional repressor of alpha-fetoprotein gene. Sci. Rep. 5, 11979 (2015).

40. Liu, X. et al. Zinc finger protein ZBTB20 promotes Toll-like receptor-triggered innate immune responses by repressing IkappaBalpha gene transcription. Proc. Natl Acad. Sci. USA 110, 11097-11102 (2013).

41. Zhou, G. et al. Zbtb20 regulates the terminal differentiation of hypertrophic chondrocytes via repression of Sox9. Development 142, 385-393 (2015).

42. Li, H. et al. SirT1 modulates the estrogen-insulin-like growth factor-1 signaling for postnatal development of mammary gland in mice. Breast Cancer Res. 9, R1 (2007).

\section{Acknowledgements}

We thank Dr A. F. Parlow for providing the hormone antibodies. This work was supported by the grants from National Natural Science Foundation of China (81130084, 31201086, 31270814, and 31470759), National Key Basic Research Program (2012CB524904 and 2013CB530603) and Shanghai Natural Science Foundation (12ZR1436900).

\section{Author contributions}

D.C. and W.Z. designed the research. D.C., X.M., J.C., J.L., Y.C., R.C., A.L., and R.Y. performed the research. M.G. contributed to the reagent. D.C., Z.X. G.S., Y.S., D.Z., X.C., M.G., and W.Z. analysed the data. D.C and W.Z. wrote the paper. W.Z. and Z.X. contributed to the study supervision.

\section{Additional information}

Supplementary Information accompanies this paper at http://www.nature.com/ naturecommunications

Competing financial interests: The authors declare no competing financial interests.

Reprints and permission information is available online at http://npg.nature.com/ reprintsandpermissions/

How to cite this article: Cao, D. et al. ZBTB20 is required for anterior pituitary development and lactotrope specification. Nat. Commun. 7:11121 doi: 10.1038/ ncomms11121 (2016)

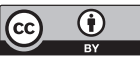

This work is licensed under a Creative Commons Attribution 4.0 International License. The images or other third party material in this article are included in the article's Creative Commons license, unless indicated otherwise in the credit line; if the material is not included under the Creative Commons license, users will need to obtain permission from the license holder to reproduce the material To view a copy of this license, visit http://creativecommons.org/licenses/by/4.0/ 